\title{
Summations over Equilaterally Triangulated Surfaces and the Critical String Measure*
}

\author{
Dirk-Jan Smit ${ }^{\star \star}$
}

Department of Physics, University of California, Berkeley, CA 94720, USA and Theoretical

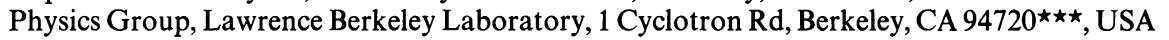

Received October 4, 1990

\begin{abstract}
We propose a new approach to the summation over dynamically triangulated Riemann surfaces which does not rely on properties of the potential in a matrix model. Instead, we formulate a purely algebraic discretization of critical string path integral. This is combined with a technique which assigns to each equilateral triangulation of a two-dimensional surface a Riemann surface defined over a certain finite extension of the field of rational numbers, i.e. an arithmetic surface. Thus we establish a new formulation in which the sum over randomly triangulated surfaces defines an invariant measure on the moduli space of arithmetic surfaces. It is shown that because of this it is far from obvious that this measure for large genera approximates the measure defined by the continuum theory, i.e. Liouville theory or critical string theory. In low genus this subtlety does not exist. In the case of critical string theory we explicitly compute the volume of the moduli space of arithmetic surfaces in terms of the modular height function and show that for low genus it approximates correctly the continuum measure. We also discuss a continuum limit which bears some resemblance with a double scaling limit in matrix models.
\end{abstract}

\section{Introduction and Summary}

Recently it has become clear that sums over certain discretizations of two dimensional surfaces, referred to as dynamical triangulations (DT) may provide a suitable framework for studying (non-perturbative) 2D gravity and eventually

\footnotetext{
* This work was supported in part by the Director, Office of Energy Research, Office of High Energy and Nuclear Physics, Division of High Energy Physics of the U.S. Department of Energy under Contract DE-AC03-76SF00098

$\star \star$ Supported in part by NSF grant PHY85-15857

$\star \star \star$ Email address: SMIT @ LBL.bitnet
} 
string theory. Contrary to the case in the usual Regge calculus where one considers triangulations with variable lengths of the triangles, one now considers triangulations consisting just of flat triangles which may be chosen to be equilateral, all having the same size $[1,2]$. As a consequence, since the length is no longer a dynamical variable, the geometry of the two dimensional surface is encoded in the number of triangles meeting at each vertex. If six triangles meet at each vertex, a flat Euclidean surface is described, while a larger (smaller) number of triangles gives rise to a surface with negative (positive) scalar curvature.

Consequently, in the partition function of the bosonic string one replaces the sum over two dimensional geometries by a summation over triangulations. The discretized partition function for a string moving in $D$ dimensions reads [1]

$$
\mathscr{Z}_{g}=\sum_{G_{i j}^{(n)} \in \mathscr{T}} \frac{1}{\left|G_{i}\right|} \int \prod_{k} \frac{d^{D} X_{k}}{(2 \pi)^{D / 2}} \exp (-\mathscr{A}[X, \mathscr{T}]),
$$

where $G_{i j}^{(n)}$ denotes the adjacency matrix specifying a triangulation with $n$ vertices, in the set of $\mathscr{T}$ of all equilateral triangulations of a given genus $g$ surface, and $\left|G_{i}\right|$ denotes the order of the symmetry group of the triangulation. The term $\mathscr{A}$ corresponds to the descretized Polyakov action

$$
\mathscr{A}[X, \mathscr{T}]=\frac{1}{2} \sum_{i, j}^{n}\left(X_{i}-X_{j}\right)^{2} G_{i j}^{(n)}+\beta \sum_{i=1}^{n} \log s_{i} .
$$

The first term is the discrete analogue of $\int d^{2} \xi \sqrt{g}(\nabla X(\xi))^{2}$ in the continuum formulation of the string, while the terms in the second sum correspond to curvature terms of which the first is the Euler characteristic expressed in terms of the vertices, while the second is the analogue of $-\beta \int d^{2} \xi \sqrt{g} R^{2}(\xi)$. (This term is irrelevant in the continuum theory.) We refer to [1] for further details on the notation. Integrating out the $X_{i}$ fields, with $\beta=0$, the partition function becomes

$$
\mathscr{Z}_{g}=\sum_{G_{i j}^{(n)}}\left(\operatorname{det}\left(s_{i} \delta_{i j}-G_{i j}^{(n)}\right)\right)^{-D / 2},
$$

where $s_{i}$ denotes the discrete surface element.

Formally this amounts to a discretization of the integral over the space of metrics, after appropriate gauge fixing, in the continuum formulation of the theory. One would like to make contact with an apparently different approach to $2 D$ gravity namely Liouville theory, in which one constructs an invariant measure on the space of all two dimensional metrics after appropriate gauge fixing. In some sense one would like to argue that the above expression defines a discretization of this measure. Of course, (1.3) defines an invariant measure on the space of all two dimensional triangulations of a surface (independent of the metrical properties of the dynamical triangulations). The popular belief is that in the continuum limit this measures agrees with the one from Liouville theory. There is, however, a subtle paradox here which prevents us from concluding that the two approaches to $2 d$ gravity are indeed two sides of the same coin. This puzzle is present in any embedding dimension $D$, hence also in critical string theory. The puzzle arises due to the fact that dynamically triangulated surfaces are badly distributed in the moduli space of a generic genus $g$ Riemann surface. Its solution would reveal either a striking property of Liouville theory and critical string theory which becomes 
only visible in high genus, or would render an inconsistency between the DT approach and the continuum formulation of (sub-)critical string theory.

One of the main results of this paper is to explain explicitly how this paradox arises as a consequence of an algebraic property of the DT approach. For this we will present an alternative formulation of the DT approach which does not rely on properties of a matrix model. It applies essentially to any embedding dimension $D$ and includes the description using matrix models. In this formulation the sum over triangulations in (1.1) corresponds to a replacement of the original of the continuum integral over moduli space $\mathscr{M}_{g}$ by a discrete sum over special points in the moduli space corresponding to so-called arithmetic surfaces. Such a replacement in case of the critical bosonic string has been proposed already in $[4,5]$. The weights attached to each triangulation, i.e. to each algebraic point in $\mathscr{M}_{g}$, is the symmetry factor $\left|G_{i}\right|$ in (1.1). As generic triangulations do not have any symmetries this factor is generically unity in the DT approach. It is far from obvious, however, that with this weight (1.3) will reproduce the string measure obtained from the continuum theory. The problem is, as was discussed also in [4], that arithmetic surfaces are for high genus $(g>23)$ badly distributed in $\mathscr{M}_{g}$. We emphasize that this problem exists irrespective of the dimension $D$, and becomes manifest only for high genus, i.e. for large orders in perturbation theory. In this paper we will discuss this problem for critical bosonic string theory, where explicit computations are not so difficult. For low genus the problem does not exist as we will verify explicitly in the case of critical string theory.

In order to appreciate the fundamental difference between the DT approach and the continuum theory let us briefly discuss the familiar interpretation of (1.3). For this it turns out to be useful to pass to the dual lattice of the triangulation. In terms of the dual lattice, the partition function is seen to correspond to the free energy of a matrix field theory with a Gaussian propagator [1,2]. In such a theory one considers an $N \times N$ Hermitian matrix field $\Phi(x)$ with action

$$
\begin{aligned}
S[\Phi]= & -\frac{1}{2} \operatorname{Tr}\left(\int d^{D} x d^{D}(y) \Phi(x) e^{(1 / 2)(x-y)^{2}+\beta} \Phi(y)\right) \\
& +\frac{N}{(2 \pi)^{-D / 2}} \operatorname{Tr}\left(\int d^{D} v V(\Phi(x))\right)
\end{aligned}
$$

where $V(\Phi)$ is the potential

$$
V(\Phi)=g_{1} \Phi+\frac{1}{2} g_{2} \Phi^{2}+\frac{1}{3} g_{3} \Phi^{3}+\cdots
$$

The $1 / N^{2}$ expansion of the free energy is related to perturbation expansion of the partition function (1.1) by

$$
\mathscr{Z}=\sum_{g=0}^{\infty} N^{-2 g-2} \mathscr{Z}_{g}(\beta)+\text { const. }
$$

Expression (1.3) makes sense in principle in any dimension, but so far it has only been practical in dimensions $D \leqq 1$, i.e. for sub-critical string theory. One finds that the spectrum of a bosonic string in $D \leqq 1$ can be obtained from Feynman diagram techniques for the propagator and vertices in the matrix theory. The surprising fact is that in this region the matrix theory has been shown to have an interesting continuum limit in which $N \rightarrow \infty$ in a subtle way, the so-called double scaling limit, which shows the existence of a phase transition of the theory, allowing 
one to consider the theory beyond the asymptotic expansion [3]. This is very different from Liouville theory which is strictly perturbative and for which so far the sum over all genera is meaningless.

In this paper we address the problem of relating the continuum critical string theory with the DT approach, taking a different approach towards (1.1) which does not rely on a matrix model. Instead, we will use an apparent different formulation of discretizing the string path integral proposed in $[4,5]$ in combination with a recent mathematical result by Voedvodski and Shabat in [6]. In short, the result in [6] states that every equilateral triangulated surface corresponds in a one-to-one way with an algebraic point in moduli space. (An algebraic point in moduli space corresponds to an arithmetic surface which is specified by a polynomial equation for which the coefficients lie in an algebraic number field, i.e. a finite extension of the rationals Q.) Varying the number of vertices corresponds with varying the number field. In this way we establish a new, alternative description of DT approach, in which each graph of given topology is in a one-to-one correspondence with an arithmetic surface of the same topology. This new formulation is suitable to discuss the above mentioned problem.

Applied to critical string theory we shall show that the summation over equilateral triangulations in (1.3) equals the volume of the lattice in $\mathscr{M}_{g}$ formed by the algebraic points computed with the string measure. The volume of this lattice was computed earlier in $[7,8]$ and is given by the so-called modular height function on $\mathscr{M}_{g}$. As was shown there, the density of the string measure with respect to the canonical measure equals the inverse archemedean part of the modular height function of an algebraic point in the moduli space. Along these lines we establish a formulation of the critical string measure in terms of the combinatorial data of the equilateral triangulation of a genus $g$ surface. Such an approach has been advocated earlier by Manin in [9].

For low genus our expression indeed converges to the familiar expression for the string path integral. However, for high genera this is far from obvious. The number of arithmetic surfaces for a finite extension $K$ of $\mathbf{Q}$, i.e. the number of $K$-rational points in $\mathscr{M}_{g}$ are contained in a low dimensional variety denoted by $\mathscr{A}_{g}$ of $\mathscr{M}_{g}$. Although the closure $\tilde{A}_{g}$ of the space $\mathscr{A}_{g}$ (corresponding to the $\overline{\mathbf{Q}}$ algebraic points of $\mathscr{M}_{g}$ ) coincides with $\overline{\mathscr{M}}_{g}$ (the compactified moduli space) the $\overline{\mathbf{Q}}$ algebraic points are very badly distributed in $\mathscr{M}_{g}$. That is, the sum in (1.1) which is in fact defined on $\overline{\mathscr{A}}_{g}$, is peaked at the points corresponding to finite extensions. ${ }^{1}$ A priori there is no indication that the measure obtained from the continuum theory is peaked at algebraic points which for any finite extension form a subspace of finite codimension in $\mathscr{M}_{g}$. It would therefore be interesting to see whether the computations done in the DT approach are in some special gauge of the continuum theory in which the measure gets its support only from the algebraic points thereby resolving the paradox. One way to study this is to investigate Liouville theory or critical string theory for high genus Riemann surfaces.

In [10] previous attempts have been made, based on techniques developed by 't Hooft [31] to assign a given triangulation to a metric on a Riemann surface,

\footnotetext{
${ }^{1}$ The Euler numbers of $\overline{\mathscr{A}}_{g}$ and $\overline{\mathscr{M}}_{g}$ are the same: the difference lies in the geometry of the two spaces
} 
or more precisely, a conformal equivalence class of metrics. In the sum over triangulations one has to control the density of vertices which turned out to be very difficult if not impossible. So these attempts could not provide a successful description of the spectrum of string in terms of the combinatorial data of the triangulation, a problem essentially already discussed in [31]. The result in [6] provides more information: namely the number field over which the Riemann surface is defined. The degree of the extension of this number field turns out to be a measure for the density of vertices. We will show how to incorporate this extra information such that one may overcome the above mentioned problem.

To discuss the summation over all $\overline{\mathbf{Q}}$ algebraic points we construct a continuum limit which turns out to be, at least qualitatively, similar to the double scaling limit in a matrix theory. It is clear from the above that such a limit is very subtle. As will be shown, for this one needs to introduce an extra parameter which upon fine tuning allows one to take the limit to $\overline{\mathbf{Q}}$.

The paper is organized as follows. First we discuss a discretization of the measure on $\mathscr{M}_{g}$ as proposed earlier in $[4,5]$. This will be done in Sect. 2 where we will use intersection theory to show that this discretization depends on the modular height function. This section is in fact a reformulation of the results in $[4,5,8,7]$ put in a different context, more suitable for discussing a possible "continuum limit."

Subsequently, in Sect. 3 we discuss the relation with equilaterally triangulated surfaces. First we will explain briefly the result of [6] and provide some explicit examples of their result. From this it will follow that (1.1) in fact defines a measure on the space of arithmetic surfaces. We will then establish a relation between certain triangulations and the geometry of the moduli space of genus $g$ using the so-called accessory parameters for $n$-punctured spheres. By a result in [13] the accessory parameters are related with the Weil-Petersson Kähler form on the moduli space of an $n$-punctured sphere.

In Sect. 4 we will use this to discuss a continuum limit necessary to define (1.3) and relate it to the discretization described in Sect. 2. As we will argue this then implies that the volume of the moduli space of curves of genus $g$ measured by the critical bosonic string measure is for low genus given by the height function summed over the rational points up to a finite rational constant.

After this work was done we were informed by $P$. Nelson that in the same spirit as in $[4,5,9]$ ) the authors of ref. [11] made an attempt also to use the result [6] in the context of $2 D$ gravity essentially running into the same paradox mentioned above. Some of the discussion presented there has been previously discussed in [4].

\section{A Discretization of the Polyakov Measure}

In this section we will construct a discretization of the Polyakov measure using a height function. Such a function turns out to be a generalization of a Green's function which is used to put a hermitian metric on determinant line bundles. The discretization discussed in this section looks conceptually quite different from the discretization in [3]. In later sections we shall see, however, that there is deep relation between the two. 
In the first part of this section we will discuss the relevant Green's functions and discuss properties of the metrics associated with it. Subsequently, we use some intersection theory to explain the role of the height function. The main purpose of this section is to explain the derivation of (2.41), which appeared earlier in $[4,5,7,8]$, and to put this result in a different perspective. The formalism used in this section is explained in more detail in older work on the mathematical formulation of bosonic string theory $[16,15,17]$.

2.1. The Definition of the Polyakov Measure. In the Friedan-Shenker approach to perturbative string theory [15] the partition function of the string corresponds to a Hermitian metric on the tensor product of a holomorphic determinant line bundle and some holomorphically (projectively) flat vector bundle on the moduli space of stable Riemann surfaces of genus $g$. In the simplest case, corresponding to the usual formulation of the bosonic string, this vector bundle is a determinant line bundle as well. Since all such line bundles are by a theorem by Harer isomorphic to the determinant line bundle of holomorphic differentials on the Riemann surface, it is not so difficult to describe this metric in this case. It arises upon using two fundamental isomorphisms. The first one is the so-called Kodaira-Spencer isomorphism. Let

$$
\pi: X \rightarrow \mathscr{M}
$$

be the universal curve over the moduli space $\mathscr{M}$ defined over the integers $\mathbf{Z}$. (To be more precise: $\mathscr{M}$ is the moduli stack over $\mathbf{Z}$ of stable curves of genus $g$.) Associated with it we have the relative dualizing sheaf ${ }^{2} \omega_{X / \mathscr{M}}$ on the Riemann surface $X$. (By abuse of notation we refer to the fibers of $\pi$ as Riemann surfaces $X$ ). The Kodaira-Spencer isomorphism is a map of the square of the dualizing sheaf and the cotangent bundle of moduli space (i.e. the bundle of holomorphic quadratic differentials on $X$ ):

$$
\left(\operatorname{det} \pi_{*}\right) \omega_{X / \mathscr{M}} \cong \operatorname{det} \Omega_{\mathscr{M} / \mathbf{Z}} \otimes \mathcal{O}(\Delta),
$$

where $\Delta$ denotes the compactification divisor of the moduli space in the Deligne-Mumford compactification [18]. We refer for the notation to [7, 4]. The second isomorphism we need is the so-called Mumford-isomorphism [19]

$$
\left(\operatorname{det} \pi_{*}\right) \omega_{X / \mathscr{M}}^{2} \cong\left(\operatorname{det} \pi_{*} \omega_{X / M M}\right)^{\otimes 13} \otimes \mathcal{O}(-\Delta), \quad g>1 .
$$

Mumford has shown that $\left(\operatorname{det} \pi_{*} \omega_{X / \mathscr{M}}\right)^{\otimes 13}$ is in fact a holomorphically trivial bundle. Combining (2.2-3) one finds the isomorphism $\mu$,

$$
\left(\operatorname{det} \pi_{*} \omega_{X / \mathscr{M}}\right)^{\otimes 13} \stackrel{\mu}{\cong} \operatorname{det} \Omega_{\mathscr{M} / \mathbf{Z}} \otimes \mathcal{O}(2 \Delta), \quad g>1 .
$$

This isomorphism is unique up to a multiplicative constant, depending only on the genus and which corresponds physically to the string coupling constant.

The Polyakov measure on $\mathscr{M}_{g}$ is constructed out of (2.4) by introducing Hermitian metrics on the left-hand side of (2.4) such that (2.4) becomes an isometry. Although differently formulated, the isomorphism (2.4) is at the origin of the famous Belavin-Kniznik theorem [20].

${ }^{2}$ Loosely speaking this is the bundle of holomorphic differentials 
A suitable metric on the bundle of holomorphic one-forms is provided by a Green's function on $X$. For convenience we recall in this context a lemma of Arakelov.

Lemma [21]. Let $X$ be a Riemann surface and let $P$ be a point on $X$. Let $d s^{2}$ be an arbitrary but given Hermitian metric on $X$, with volume form $d \mu$. Then there is a unique function $G(P, z)$ of $z \in X$ having the following properties:

1. $G(P, z)$ is a smooth non-negative real-valued function of $z$ which has a unique first order zero at $z=P$;

2.

$$
\left.\partial_{z} \bar{\partial} \log G(P, z)\right|_{z=P}=\left.i \pi\left(d \mu-\delta_{P}\right)\right|_{z=P},
$$

where $\delta_{P}$ is the $(1,1)$ current representing the evaluation of $(0,0)$ forms at $z=P$. That is

$$
\int_{X} f(z) \delta_{P}=f(P)
$$

3.

$$
\int_{X} \log G(P, z) d \mu=0
$$

4.

$$
G(P, z)=G(z, P) \text { for all } z \text { and } P \text { on } X .
$$

The function $g(P, z)=\log (P, z)$ is called the Green's function on $X$ with respect to $d \mu$.

It turns out to be useful to introduce a special Hermitian metric $d s^{2}$, called the Arakelov metric. This is only a matter of convenience: all the results turn out to be independent of the choice of the metric $d s^{2}$. The Arakelov metric is defined as follows. For a given line bundle $L$ on $X$ with a given Hermitian metric on it one has the curvature two-form:

$$
R_{L}=\partial_{z} \bar{\partial}_{z} \log |s|^{2} d z \wedge d \bar{z} . \quad s \in L,
$$

with $z$ a local coordinate. This curvature form satisfies:

$$
\int_{X} R_{L}=2 \pi i \operatorname{deg}(L)
$$

with the integer $\operatorname{deg}(L)$ denoting the degree of $L$. One calls $L$ an admissible line bundle with respect to the Hermitian metric $d s^{2}$ on $X$ if

$$
R_{L}=2 \pi i \operatorname{deg}(L) d \mu .
$$

Arakelov showed that each line bundle $L$ on $X$ has a unique (up to multiplication by a constant) admissible metric with respect to the metric $d s^{2}$ on $X$.

Furthermore, each admissible metric is related to the Green's function with respect to $d s^{2}$ as follows. Let $Q$ be a point on $X$. The constant function 1 is a unit section of the structure sheaf $\mathcal{O}_{X}(Q)$ (i.e. the line bundle of meromorphic functions on $X$ evaluated at $z=Q$.) One puts a Hermitian metric $\|\cdot\|$ on $\mathcal{O}_{X}(Q)$ by putting

$$
\|1\|_{\mathcal{O}_{X}}(P)=G(P, Q)=\exp g(P, Q),
$$


where the norm of the unit section is 1 evaluated at the point $P \in X$. Note that the right-hand side vanishes as $P \rightarrow Q$, which implies that this a non-singular metric on all of $X$. Property (2) implies that it is also an admissible metric. Upon taking tensor powers one may generalize this construction to obtain an admissible metric on any line bundle $\mathcal{O}_{X}(D)$ for any divisor $D$ on $X$, by taking

$$
\mathcal{O}_{X}\left(D_{1}+D_{2}\right) \cong \mathcal{O}_{X}\left(D_{1}\right) \otimes \mathcal{O}_{X}\left(D_{2}\right)
$$

to be an isometry. The metric on $\mathcal{O}_{X}(D)$ obtained in this way is referred to as the Green's metric $\|\cdot\|_{G}$.

Let us next apply this to the bundle of holomorphic differentials. We take as the metric on the space of sections of $\omega_{X / \mathscr{M}}$ :

$$
\left\langle\omega_{1}, \omega_{2}\right\rangle=\frac{i}{2} \int_{X} \omega_{1} \wedge \bar{\omega}_{2}, \quad \omega_{1,2} \in \Gamma\left(X, \omega_{X / \mathscr{M}}\right) .
$$

Fix a basis $\omega_{i}, i=1, \ldots, g$ of $\Gamma\left(X, \omega_{X / \mathscr{M}}\right)$ and choose a Hermitian metric $d s^{2}$ on $X$ such that its volume form $d \mu$ satisfies

$$
d \mu=\frac{i}{2 g} \sum_{i=1}^{g} \omega_{i} \wedge \bar{\omega}_{j}
$$

Now choose a base point $P_{0}$ on $X$, then one has the embedding

$$
P \rightarrow\left(\int_{P_{0}}^{P} \omega_{1}, \ldots, \int_{P_{0}}^{P} \omega_{g}\right)
$$

of $X$ into its Jacobian $\mathscr{J}(X) \cong \mathbf{C}^{g} / \Lambda$ with $\Lambda$ denoting the period lattice of $X$. The canonical metric on $\mathbf{C}^{g}$ induces a flat metric on $\mathscr{J}(X)$, having volume form $g d \mu$. We will use this fact to introduce an admissible metric on $\omega_{X / \mathscr{M}}$ with respect to $d s^{2}$ defined as above. Namely, let $\mathcal{O}_{X}(P)$ have its Green's metric (2.12), where the Green's function is with respect to the metric $d s^{2}$ on $X$. The residue of a differential at $P$ gives an isomorphism of the fibre at $P$ of

$$
\omega_{X / \mathscr{M}}(P) \cong \omega_{X / \mathscr{M}} \otimes \mathcal{O}_{X}(P)
$$

to C. Now, give $\omega_{X / \mathscr{M}}$ the Hermitian metric such that for all points $P$ this residue becomes an isometry. This defines a Green's metric on $\omega_{X / \mathscr{M}}$ with respect to $d s^{2}$. Explicitly:

$$
\|s\|_{G}(Q)=\lim _{P \rightarrow Q} \frac{\left|z_{P}-z_{Q}\right|}{G(P, Q)}, \quad s \in \omega_{X / \mathscr{M}},
$$

which is indeed an admissible metric on $\omega_{X / \mathscr{M}}$. The metric $d s^{2}$ has volume form

$$
d \mu=\frac{1}{4 \pi(1-g)} R
$$

If we had not chosen this metric but instead an arbitrary Hermitian metric then the Green's metric would not be so simply related with the canonical metric on the Jacobian.

There exists a natural way to transfer the admissible metric on $\omega_{X / \mathscr{M}}$ to a metric on the determinant line bundle $\operatorname{det} \pi_{*} \omega_{X / \mathscr{M}}$. The metric thus obtained is 
referred to as the Faltings metric and denoted as $\|\cdot\|_{F}$. We refer the reader to [22, Theorem 1] for a formulation of this fact. The theorem implies that the Faltings metric on $\left(\operatorname{det} \pi_{*} \omega_{X / \mathscr{M}}\right)^{\otimes 13}$ can be expressed in terms of theta functions [23]. ${ }^{3}$ An important property of the Faltings metric is that the isomorphism (2.4) is an isometry for this metric. This follows from a local version of the GrothendieckRiemann-Roch theorem as formulated in [26]. For details on this theorem and its application to the Faltings metric we refer to $[17,4]$. Thus we obtain a metric on (det $\Omega_{\mathscr{M} / \mathbf{Z}} \otimes(2 \Delta)$. Passing to the dual bundle we obtain a Hermitian metric on the determinant line bundle det $T_{\mathscr{M} / \mathbf{Z}}$ the tangent bundle to $\mathscr{M} / \mathbf{Z}$. In $[16,4]$ it is shown that this metric up to a multiplicative constant depending only on the genus is the Polyakov measure. It reads explicitly

$$
\frac{\mu(s) \wedge \overline{\mu(s)}}{\|s\|^{2}}=\prod_{i=1}^{3 g-3} \phi_{i} \wedge \bar{\phi}_{i}\left\|1_{\theta}\left(P_{1}, \ldots, p_{3 g-3}\right)\right\|^{2} \cdot C
$$

with

$$
C_{p} \equiv \lambda_{g} \exp (9 \delta / 4) \frac{\prod_{i \neq j} G\left(P_{i}, P_{j}\right)}{\mid \operatorname{det}\left(\left.\phi_{i}\left(P_{j}\right)\right|^{2}\right.},
$$

where $\phi_{i}$ denotes a holomorphic quadratic differential and $\delta$ denotes Faltings' invariant on a Riemann surface [22], and $\mu$ is the isomorphism (2.4). Furthermore, $\lambda_{g}$ is a genus dependent parameter. In (2.20) $s$ is a section of $\left(\operatorname{det} \pi_{*} \omega_{X / \mathscr{M}}\right)^{\otimes 13}$. The metric on this bundle is the metric defined in (2.15).

2.2. From Green's Functions to Intersection Theory. The formulation of the partition function as a metric using Green's functions is suitable for a generalization to arithmetic surfaces, i.e. compact Riemann surfaces defined over some algebraic number field. This generalization essentially relies on the introduction of a suitable intersection product of divisors on the arithmetic surface which, for the number field $\mathbf{C}$, coincides with the Green's function $\log G(P, z)$ introduced in the previous subsection. We start off with the definition of the so-called Arakelov intersection product on arithmetic surfaces. Subsequently we will describe the relation between this intersection product and a height function. We will be brief here, as most of the mathematics is described in $[4,5]$.

An arithmetic surface is loosely speaking the analogue of a Riemann surface defined over an algebraic number field $K$, (i.e. a finite extension of $\mathbf{Q}$ ). In general it is given by a set of polynomial equations (like "ordinary" curves), but unlike the usual situation, the coefficients and solutions are elements in some'algebraic number field $K$. The mathematically correct way to describe such surfaces involves

${ }^{3}$ Originally, the string partition function was shown to correspond to a different metric, the so-called Quillen metric, [24]. The chiral bosonization formula in [25, 17] (which provided an alternative way to proving that the partition function is expressible in terms of theta functions), are equivalent due to the following relation between the Faltings metric and the Quillen metric:

$$
\|\cdot\|_{F}=\Lambda_{g}\left(\frac{\operatorname{det}^{\prime} \Delta_{0}}{A}\right)^{-1 / 2}\|\cdot\|_{Q}
$$

where $\Lambda$ is a constant only depending on the genus, $\Delta_{0}$ is the scalar Laplacian computed with the Arakelov metric, $A$ the area of the Riemann surface and $\|\cdot\|_{Q}$ the Quillen metric. (See e.g. [4].) 
a so-called affine projective scheme. That is, one describes the arithmetic surface as

$$
\pi: X \rightarrow \operatorname{Spec}\left(\mathcal{O}_{K}\right),
$$

where $\mathcal{O}_{X}$ denotes the ring of integers of $K$. This defines an affine surface, hence we need a suitable compactification of $\pi$ in order to get a precise analogy with a compact Riemann surface. This is done by considering the infinite places of $K$ which arise upon the embeddings of $K$ into the complex numbers. There are exactly $[K: \mathbf{Q}]$ of these embeddings. (The notation $[K: \mathbf{Q}]$ denotes the degree of the extension of $K$.) The fibers of $\pi$ above the infinite places of $K$ define a compactification of the arithmetic surface: each fibre over an infinite place is isomorphic to a compact Riemann surface. Thus $X\left(K_{v}\right) \cong X(\mathbf{C})$, for each infinite place $v$. From now on we assume that there is a Hermitian metric $d s_{\infty}^{2}$ given on the Riemann surface $X\left(K_{v}\right)$ for each infinite place $v \in M_{K}^{\infty}$. We have the following commutative diagram:

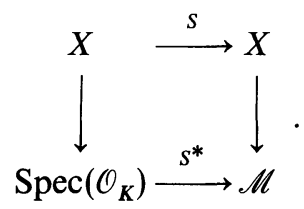

On the compact surface $X$ one may introduce an Arakalov divisor $D$ which is defined as

$$
D=D_{\text {fin }}+D_{\text {inf }}=\sum_{v \in M_{K}^{\text {fin }}} k_{v} C_{v}+\sum_{v \in M_{K}^{\infty}} \lambda_{v} F_{v},
$$

where $k_{v}$ are integers and the $C_{v}$ denote the fibers of $\pi$ over the finite places $v \in M_{K}^{\mathrm{fin}}$. In other words

$$
C_{v}=\pi^{-1} \operatorname{Spec}\left(\mathbf{F}_{v}\right)
$$

where $\mathbf{F}_{v}$ denotes the residue class field of $K$. Its order (i.e. the number of elements) is denoted by $q_{v}$. In the second term in (2.24) the symbol $F_{v}$ stands for the fibre of $\pi$ over the infinite places $v \in M_{K}^{\infty}$ of $K$. The $\lambda_{v}$ are real numbers.

The main result of ref. [21] we need here is the construction of an intersection product for divisors $D$ which at the infinite places is given by a Green's function with respect to the metric $d s_{\infty}^{2}$ on the Riemann surface $X\left(K_{v}\right)$ for each infinite place $v$. The intersection product $\langle$,$\rangle is defined as follows:$

$$
\left\langle D_{1}, D_{2}\right\rangle \equiv\left\langle D_{1}, D_{2}\right\rangle_{\text {fin }}+\left\langle D_{1}, D_{2}\right\rangle_{\text {inf }},
$$

where $\left\langle D_{1}, D_{2}\right\rangle_{\text {fin }}$ is the intersection product of two Arakelov divisors $D_{1}, D_{2}$ on $X$ over the finite places. This quantity is given by

where

$$
\left\langle D_{1}, D_{2}\right\rangle_{\mathrm{fin}}=\sum_{v \in M_{\mathrm{K}}^{\mathrm{fin}}}\left\langle D_{1}, D_{2}\right\rangle_{v},
$$

$$
\left\langle D_{1}, D_{2}\right\rangle_{v}=\log \left(q_{v}\right) \times(\text { the intersection multiplicity). }
$$

For the infinite places one has

$$
\left\langle D_{1}, D_{2}\right\rangle_{\mathrm{inf}}=\sum_{v \in M_{\mathrm{K}}^{\infty}}\left\langle D_{1}, D_{2}\right\rangle_{v}
$$


which is related to the Green's function on $X\left(K_{v}\right) \cong X(\mathbf{C})$ with respect to $d s_{\infty}^{2}$ :

$$
\left\langle D_{1}, D_{2}\right\rangle_{v}=\varepsilon_{v} \log G\left(D_{1}, D_{2}\right), \quad v \in M_{K}^{\infty},
$$

where $\varepsilon_{v}=1(2)$ for $K_{v}=\mathbf{R}(\mathbf{C})$.

Having introduced divisors and an intersection product on $X$ one can now define line bundles on $X$ which over the Riemann surface at the infinite places can be given Hermitian metrics induced from the Green's function in the way described in the previous subsection. Such line bundles will be called metrized line bundles. For an Arakelov divisor $D$ we let

$$
L=\mathcal{O}\left(D_{\text {fin }}\right)
$$

be the line bundle on $X$ which has the Green's metric on the induced bundle on $X_{v}$ for each infinite place $v$, with respect to the hermitian metric $d s_{v}^{2}$. Thus for the infinite places we have an admissible metric on the induced line bundle. Let $s$ be a section of $L$. The divisor of $s$ is defined to be

$$
\operatorname{div}(s)=(s)_{\mathrm{fin}}+\sum_{v \in M_{\mathbf{K}}^{\infty}} w_{v} F_{v}
$$

with $(s)_{\mathrm{fin}}$ the divisor of $s$ on the finite part of $X$ and

$$
w_{v}(s)=\int_{X_{v}}-\log |s|_{v} d \mu_{v}
$$

where $d \mu_{v}$ is the volume form of the Hermitian metric on $X_{v}$ for each infinite place $v$. Now, recall that admissible metrics are unique up to scalar multiplication. Thus

$$
L \cong \mathcal{O}_{X}(\operatorname{div}(s)) \text {. }
$$

We now want to relate the degree of a metrized line bundle on $X$ with the intersection product for the divisor of its sections. Let $S=\operatorname{Spec}\left(\mathcal{O}_{K}\right)$ and consider a section $s(S)$ of $\pi$ of which we let $D_{1}$ be its divisor. Let $L=\mathcal{O}_{X}\left(D_{2}\right)$ be the metrized line bundle defined by an arbitrary Arakelov divisor $D_{2}$ on $X$. From [22] we learn that for every

$$
\left\langle D_{1}, D_{2}\right\rangle=\operatorname{deg}\left(s^{*}\left(\mathcal{O}_{X}\left(D_{2}\right)\right),\right.
$$

where "deg" denotes the degree of the metrized line bundle $L$ defined as [22]

$$
\operatorname{deg}(L)=\log \left(\operatorname{order}\left(L / \mathcal{O}_{K} \cdot t\right)\right)-\sum_{v \in M_{K}^{\infty}} \varepsilon_{v} \log |t|_{v},
$$

with $t$ a section of $L$. This concludes our survey of the arithmetic calculus on $X$. We are now ready to describe a discretization of the Polyakov measure, announced in the beginning of this section.

2.3. Discretizing the Polyakov Measure Using the Modular Height Function. Recall that the Polyakov measure defines a real valued function on the Jacobian of the Riemann surface $X$. We will now show that when one considers it as an arithmetic surface this function gets replaced by a so-called height function. Let $\theta$ be the theta divisor in the Jacobian $\mathscr{J}$ of the arithmetic surface $X$. This is an Arakelov divisor and $\mathcal{O}_{X}(\theta)$ is an metrized line bundle of degree $g-1$ (we assume $g>1$ ) on $X$. The sections of this metrized line bundle give rise to the embedding of the 
Jacobian into a projective space:

$$
\phi: \mathscr{J} /( \pm 1) \rightarrow \mathbf{P}\left(H^{0}\left(\mathscr{J}, \mathcal{O}_{X}(\theta)\right)\right) .
$$

The image has degree $2^{g-1} g$ ! and the dimension of the projective space is $2^{g}-1$. Now choose an isomorphism

$$
\mathbf{P}\left(H^{0}\left(\mathscr{J}, \mathcal{O}_{X}(\theta)\right)\right) \cong \mathbf{P}^{2^{g}-1}(K)
$$

and define for a point $z=\left(z_{1}, \ldots, z_{2^{g}}\right) \in \mathbf{P}^{2 g-1}(K)$ the canonical height $h(z)$ :

$$
h(z)=\sum_{v} \max \left\{\log \left|z_{1}\right|_{v}, \ldots, \log \left|z_{2^{g}}\right|_{v}\right\},
$$

where the sum runs over all places of $K$. Note that this definition shows that the height is in fact a distance function, measuring the logarithmic distance to the closest $K$-rational point. ${ }^{4}$

A result by [27] states that for a point $\alpha$ on the Jacobian the limit

$$
h_{\mathscr{J}}(\alpha) \equiv \lim _{n \rightarrow \infty} \frac{h \circ \phi(n \alpha)}{n^{2}}
$$

exists, is independent of the sections chosen in $\mathcal{O}(\theta)$, and defines a positive real valued function $h_{\mathscr{J}}: \mathscr{J} \rightarrow \mathbf{R}$. We are interested in computing this function. In principle this can be done by using the intersection theory described above. However, to do the calculation in practice one estimates this height function by the modular height function introduced by Faltings. This function is defined as follows. Let $\pi: X \rightarrow \operatorname{Spec}\left(\mathcal{O}_{K}\right)$ be the universal curve. (See the diagram (2.23).) Then one defines:

$$
h(X)=\frac{1}{[K: \mathbf{Q}]} \operatorname{deg}\left(\operatorname{det} \pi_{*} \omega_{X / S}\right) .
$$

This is the modular height function and can be computed using Arakelov intersection theory on $X$. It is understood that the line bundle appearing on the right-hand side is a metrized line bundle. One may regard the modular height function as a distance function for the rational points on the moduli space over $K$.

Now return to the isometry (2.20) defining the Polyakov measure. It gives Hermitian metrics at the line bundle $S^{*} \operatorname{det} T_{\mathscr{M} / \mathbf{Z}}$. The $\mathcal{O}_{K}$-module defines a lattice in

$$
S^{*} T_{\mathscr{M} / \mathbf{Z}} \otimes \mathbf{Z} \mathbf{R}=\prod_{v \in M_{K}^{\infty}} S_{v}^{*} T_{\mathscr{M} / \mathbf{Z}}
$$

on which the Polyakov measure defines a volume form. In [7,8] the volume of $\left(S^{*} T_{\mathscr{M} / \mathbf{Z}} \otimes_{\mathbf{Z}} \mathbf{R}\right) / S^{*} T_{\mathscr{M} / \mathbf{Z}}$ has been computed using the Riemann-Roch theorem on $\operatorname{Spec}\left(\mathcal{O}_{K}\right)$. We will not repeat this calculation here, as it is explicitly shown there.

${ }^{4}$ This becomes more clear if we consider the height in the two-dimensional projective space $\mathbf{P}^{2}(\mathbf{Q})$. A point $z \in \mathbf{P}^{2}(\mathbf{Q})$ corresponds to a line through the origin in a three dimensional space. The height $h(z)$ measures the logarithmic distance to the origin of the closest non-zero integral lattice point (i.e. a point with integer coordinates) on this line:

$$
h(z)=\log \sqrt{a^{2}+b^{2}+c^{2}},
$$

with $(a, b, c)$ a lattice point on the line specified by $z$ 
The result reads (for genus $g>1$ )

$$
\operatorname{Vol}\left(\left(\prod_{v \in M_{\mathbf{K}}^{\infty}} S_{v}^{*} T_{\mathscr{M} / \mathbf{Z}}\right) / S^{*} T_{\mathscr{M} / \mathbf{Z}}\right)=C \cdot\left(2^{-r_{2}} D^{1 / 2}\right)^{(3 q-3) /[K: \mathbf{Q}]} \exp (13 h(X)),
$$

where the constant $C$ depends only on the number field $K$,

$$
C=N_{K / \mathbf{Q}}\left(\Delta_{X}\right)^{-2 /[K: \mathbf{Q}]} \text {. }
$$

We also have computed explicitly the height function $h(X)$ for genus 1 [28] and $2[4,5])$ :

$$
\begin{aligned}
& h(X)_{g=1}=\frac{1}{12[K: \mathbf{Q}]}\left(\log \left|N_{K / \mathbf{Q}}\left(\Delta_{X}\right)\right|-\sum_{v \in M_{K}^{\infty}} \varepsilon_{v} \log \left(\left|\Delta\left(\tau_{v}\right)\right|\left(\operatorname{Im}\left(\tau_{v}\right)\right)^{6}\right),\right. \\
& h(X)_{g=2}=\frac{1}{10[K: \mathbf{Q}]}\left(\log \left|N_{K / \mathbf{Q}}\right|\left(\Delta_{X}\right)-\sum_{v \in M_{K}^{\infty}} \varepsilon_{v} \log \left(\left|\chi_{10}\left(\tau_{v}\right)\right| \operatorname{Im}\left(\tau_{v}\right)\right)^{5}\right),
\end{aligned}
$$

which thus shows that each infinite place the height reproduces the well known formula for the string partition function [29].

One would now like to replace the original integral over the moduli space for arbitrary genus by a summation over the rational points corresponding to curves defined over $\operatorname{Spec}\left(\mathcal{O}_{K}\right)$ in the limit to the algebraic closure $\overline{\mathbf{Q}}$. That is, one would like to dedine the discrete partition function as

$$
\mathscr{Z}=\lim _{\mathbf{K} \rightarrow \overline{\mathbf{Q}}} \sum_{X / K} \operatorname{Vol}\left(\left(S^{*} T_{\mathscr{M} / \mathbf{Z}} \otimes_{\mathbf{Z}} \mathbf{R}\right) / S^{*} T_{\mathscr{M} / \mathbf{Z}}\right),
$$

where the sum is over all the rational curves defined over $K$. The rational points in moduli space form a discrete set, forming the lattice (2.40). By taking large enough extensions one may hope that the volume of this lattice reproduces the path integral over the moduli space in the continuum formulation of the bosonic string. (The $\overline{\mathbf{Q}}$ points are dense in $\mathscr{M}_{g}$.) Thus (2.42) corresponds to a "lattice approximation" of critical string theory, which has been proposed already in $[4,5]$. In the next section we shall see that each term in (2.42) in fact corresponds with a triangulated Riemann surface. Using this we will argue that (2.42) and the discretization (1.3) as defined in the DT approach are the same. The question whether (1.3) reproduces critical string theory thus becomes an arithmetic problem, involving the distribution of rational points in moduli space. As was already mentioned, it is far from obvious that this discretization will approach the continuum measure for generic genera.

It should be realized that $(2.42)$ combines both the arithmetic and complex analytical properties of the theory. Formula (2.35) for the degree of a line bundle suggests a factorization of the (2.42) like one usually has in $p$-adic analysis.

\section{Algebraic Points and Equilateral Triangulations}

The algebraic points in moduli space have a nice geometrical interpretation in terms of triangulated surfaces due to a result of Voedvodski and Shabat [6], which 
we will explore below to show that the sum in (1.3) is related to the discretization discussed in the previous section. The fundamental result is that each Riemann surface that can be triangulated by Euclidean equilateral triangles is algebraic and vice versa: to each equilateral triangulation one can assign a unique complex structure on a two dimensional surface, turning it into a Riemann surface. This Riemann surface is necessarily an arithmetic surface. This implies that the original integral over metrics in the partition function of string theory can be replaced by the summation (1.3) over triangulated surfaces corresponding to algebraic points. For this we discuss the result in [6] in some detail and develop a technique by which one may relate a given triangulation with a $K$-rational point in moduli space in some simple examples.

3.1. Triangulations. Let us begin with presenting a geometrical interpretation of an algebraic point in $\mathscr{M}$. The starting point is a theorem by Belyi [30]. It states that a complete algebraic curve $X$ over a field of characteristic zero (e.g. C), can be defined over $\overline{\mathbf{Q}}$ if and only if there exists a covering map

$$
\phi: X \rightarrow \mathbf{P}^{1}
$$

which is holomorphic outside three points (e.g. $\{0,1, \infty\}$ ).

In practice it is not so easy to find examples of such functions. In [6] it is shown that such functions are intimately related with functions depending on a period matrix $\tau$ and a complex coordinate which for certain special values of $\tau$ are functions on the complex plane that give rise to a polygon consisting exclusively of equilateral triangles. This polygon is holomorphically equivalent to the Riemann surface characterized by $\tau$. That is, the required function "transfers" the canonical complex structure (inherited from $\mathbf{C}$ ) on the interior of each triangle to the polygon such that it defines a complex structure on a two dimensional surface characterized by the period matrix $\tau$. This complex structure on $X$ is called the equilateral complex structure. The result is stated in the following.

Theorem [6]. Let $X$ be a complete nonsingular complex algebraic curve. It is defined over $\overline{\mathbf{Q}}$ if and only if there exists a simplicial scheme for which $X$ is biholomorphically equivalent to the Riemann surface $X$ with the equilateral complex structure.

The proof of the theorem relies to a large extent on the construction of the equilateral complex structure. Using conformal mappings, it is explained how in principle the required Belyi function $\phi$ can be obtained. We will explain below how one obtains from a given set of equilateral triangles a unique Riemann surface of given genus. Instead of reviewing the abstract procedure by which one shows how the complex structure is obtained, we show this explicitly in an example which turns out to be useful in the rest of this section.

In order to relate the combinatorial data of a set of triangles with a compact two dimensional surface one introduces a so-called simplicial scheme $S$ which consists of a finite set $J_{0}(S)$ which will correspond to the vertices and two collections $J_{1}(S)$ and $J_{2}(S)$ consisting of respectively two-element and three-element subsets of $J_{0}(S), J_{1}(S)$, respectively $J_{2}(S)$, correspond to the set of edges, respectively faces. For consistency one requires that all two element subsets of $J_{2}(S)$ are contained in $J_{1}(S)$. Out of $S$ one then constructs a polyhedron in a real Euclidean vector space of dimension equal to the order of the set $J_{0}(S)$. $S$ is identified with the coordinate 
vectors on this vector space and the convex hulls of the images of $J_{1}(S)$ and $J_{2}(S)$ form the edges and faces of this polyhedron. Such a polyhedron is called the realization of $S$ and denoted by $|S|$.

One next introduces a flag-set $\mathscr{F}(S)$ associated with $S$ which allows one to identify triangles on the polyhedron. It is defined as the set of tuples

$$
\mathscr{F}(S)=\left\{\alpha, \beta, \gamma \in J_{0}(S) \times J_{1}(S) \times J_{2}(S) \mid \alpha \subset \beta \subset \gamma\right\} .
$$

One also introduces the projections

$$
\pi_{q}: \mathscr{F}(S) \rightarrow J_{q}(S), \quad q=0,1,2 .
$$

In order to study a complex structure on the faces of the polyhedron which extends to the edges and vertices such that $|S|$ becomes homeomorphic to a compact Riemann surface, it turns out to be useful to introduce a group action on $\mathscr{F}(S)$ which maps triangles into each other. One defines a group $C_{2}$ acting on $\mathscr{F}(S)$ by the following relations for its generators $\sigma_{p}, p=0,1,2$ for all elements $F \in \mathscr{F}(S)$ :

$$
\sigma_{0}^{2}=\sigma_{1}^{2}=\sigma_{2}^{2}=\left(\sigma_{0} \sigma_{2}\right)^{2}=1,
$$

having the following properties

1.

$$
\pi_{q}(\sigma \circ F)=\pi_{q}(F) \Leftrightarrow p \neq q ;
$$

2. $C_{2}$ must act transitively, so that the realization $|S|$ is connected;

3. there must be an element $O \in C_{2}$

such that

$$
O: \mathscr{F}(S) \rightarrow\{ \pm 1\}
$$

$$
O\left(\sigma_{q}^{\circ} F\right)=-O(F) .
$$

It is not hard to see that for a given $S$ its realization is homeomorphic to a two dimensional surface if and only if there is a unique action of $C_{2}$ on $\mathscr{F}(S)$ satisfying the above properties.

Let us pause for a moment and see how this works in a simple example. ${ }^{5} \mathrm{We}$ will build a regular tetrahedron with vertices numbered from 1 to 4 . (See Fig. 1.)

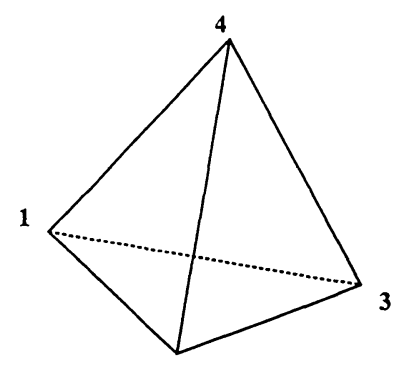

2

Fig. 1. The tetrahedron

${ }^{5}$ I thank B. Edixhoven for discussions on this point 
So we have

$$
\begin{aligned}
& J_{0}(S)=\{1,2,3,4\}, \\
& J_{1}(S)=\{\{1,2\},\{1,3\},\{1,4\},\{2,3\},\{2,4\},\{3,4\}\}, \\
& J_{2}(S)=\{\{1,2,3\},\{1,2,4\},\{2,3,4\},\{1,3,4\}\},
\end{aligned}
$$

and $S$ is realized in $\mathbf{R}^{4}$. The flagset $\mathscr{F}(S)$ is given by (3.2) and thus is of order $4 \cdot 3 \cdot 2=24$. The action of the group $C_{2}$ is easily determined from the definition of its generators. Take $F=(1,\{1,2\},\{1,2,3\}) \in \mathscr{F}(S)$, then

$$
\begin{aligned}
& \sigma_{0}(F)=(2,\{1,2\},\{1,2,3\}), \\
& \sigma_{1}(F)=(1,\{1,3\},\{1,2,3\}) .
\end{aligned}
$$

These actions are natural; we have illustrated them in Fig. 2. The action of $\sigma_{2}$ is less automatic, since it adds a new vertex to the configuration as we have, (see Fig. 3),

$$
\sigma_{2}(F)=(1,\{1,2\},\{1,2,4\}),
$$

where the new vertex is labelled by 4 . A priori, this may not be a unique vertex, however, in this example the action of $\sigma_{2}$ is unique since the order of the set

$$
\left\{\gamma \in J_{2}(S) \mid \beta \in \gamma\right\}=2 \text {. }
$$

Since all $\sigma_{i}$ change the orientation of the triangle described by $F$, the above action of $\sigma_{i}$ satisfies all the properties listed above. Observe that in addition to the relation (3.4) we have $\left(\sigma_{0} \sigma_{1}\right)^{3}=1$. This tells exactly how many triangles meet at each vertex.
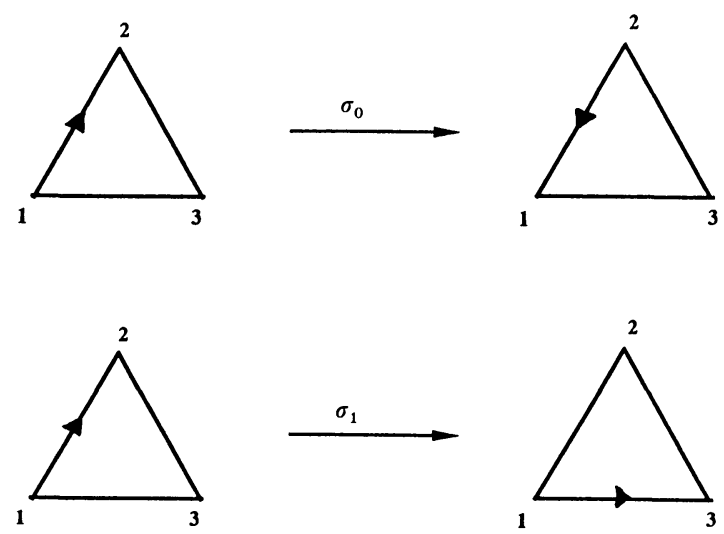

2
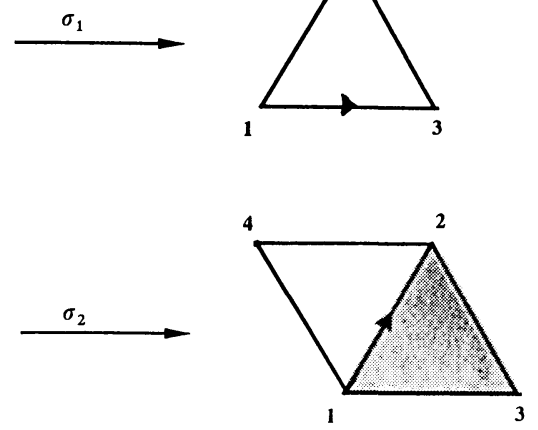

3

Figs. 2 and 3. The action of $C_{2}$ 
More generally: the orbits of the group generated by $\sigma_{i}, i=1,2$ correspond to the number of triangles meeting at each vertex. Thus we see that from a given $S$ and a flagset $\mathscr{F}$ the action of $C_{2}$ gives a unique realization $|S|$.

To show that $|S|$ is homeomorphic to a Riemann surface, requires the construction of a complex structure. In [6] such a complex structure is constructed out of the canonical complex structure on each interior of a triangle inherited from $C$. Of course, the extension to the edges and vertices will lead to a compatability condition for each of the complex structures on the faces. It turns out that there is a unique complex structure on $|S|$ which satisfies this condition.

In order to describe this complex structure one constructs a slightly different realization of $S$ in which one considers the set of triangles in the complex plane. Consider the set of equilateral triangles $T^{ \pm}$of which one side is along the interval $[0,1]$ on the real axis in the complex plane and a vertex in the upper, respectively lower, half plane. Form the disconnected set of triangles

$$
\mathscr{C}(S)=\left\{z \times F \in \mathbf{C} \times \mathscr{F}(S) \mid z \in T^{ \pm} \text {for } O(F)= \pm 1\right\} .
$$

The group $\mathscr{C}_{2}$ acts on this set so its is natural to define two equivalence relations on this set. The first equivalence relation, $R_{1}$ says that two elements in $\mathscr{C}$ are equivalent whenever $F^{\prime}=\sigma_{2} F$ and $z=z^{\prime} \in \mathbf{R}$. The other relation $R_{2}$ involves the action of $\sigma_{0}$ and $\sigma_{1}$. The group $\mathscr{C}_{1}$ generated by $\sigma_{0,1}$ acts as

$$
\begin{aligned}
& \sigma_{0}(z \times F)=\left((1-z) \times\left(\sigma_{0}(F)\right),\right. \\
& \sigma_{1}(z \times F)=\left(\left(e^{-i \mathcal{O}(F) \pi / 3}\right) \times\left(\sigma_{1}(F)\right) .\right.
\end{aligned}
$$

The quotient $\mathscr{C}(S) / R$ defined by the weakest equivalence the relation $R$ generated by $R_{1,2}$, defines a space $X(S)$ on which the following complex structure inherited from $\mathbf{C}$ can be defined.

Let $n$ be the number of triangles meeting at each vertex, i.e. for a given $F \in \mathscr{F}, n$ is the smallest integer for which $\left(\sigma_{1} \sigma_{2}\right)^{n}(F)=F$. The sum of the angles at each vertex thus equals $n \pi / 6$. Let $Z_{v}$ be a holomorphic function with respect to the canonical complex structure (inherited from $\mathbf{C}$ ) on the interior of one the $v^{\text {th }}$ triangle, $v=1, \ldots, n$. Whenever a point $P$ lies on an edge, say of the $v^{\text {th }}$ and $v+1^{\text {th }}$ triangles then

$$
Z_{v}(P)=Z_{v+1}(P) \text {. }
$$

Thus it is clear that we have holomorphic coordinates on all points except possibly at the vertices. So let $P$ be a vertex of a triangle $\Delta_{v}$. We may assume that $Z_{v}(P)=0$, and that $\Delta_{v}$ has an edge in common with the triangle $\Delta_{v+1}$. (We identify the $v$-th triangle with the $(v+1)$-th one. Let $V_{v}$ be the angle of $\Delta_{v}$ at $Z_{v}=0$. Then one may define a local coordinate $Z$ at $P$ by

$$
\left.Z\right|_{V_{v}}=Z_{v}(Q)^{\mu} e^{\mu\left(\sum V_{v}\right)},
$$

where $Q$ is point in $\Delta_{v}$ and the sum runs over all angles meeting at the origin (with $V_{0}=0$ ). The number $\mu$ is the sum of the angles: $\mu=\frac{2 \pi}{\sum V_{v}}$. Since all triangles are equilateral one may recast this into

$$
\left.Z\right|_{V_{v}}=\exp (2 \pi i v / n)\left(Z_{v}\right)^{6 / n}
$$

This turns $X(S)$ into a compact Riemann surface. In fact this formula tells us that 
each open neighborhood of a vertex in $X(S)$ with the vertex itself removed is biholomorphic to a punctured disc. The complex structure on $X(S)$ is called the equilateral complex structure. Note that when $n=6$, the triangulation describes a flat surface and the equilateral complex structure coincides to the canonical complex structure on $\mathbf{C}$. Namely, if $n=6$, rotation over 180 degrees, (that is, taking $v=3$ ) indeed corresponds to multiplication with -1 . Another important observation is that changes of the lengths of the edges of the triangles does not change the conformal class of the discretized metric.

The theorem in [6] quoted above implies that each equilateral triangulation endowed with its equilateral complex structure defines a $\overline{\mathbf{Q}}$-algebraic Riemann surface and vice versa. For us the part of the theorem

\section{Triangulation $\rightarrow$ Riemann surface}

is most important, as we shall now discuss. It follows from the existence of a Belyi function. Namely, draw the medians in each of the triangles $T^{ \pm} \in \mathscr{C}(S)$ so that one obtains 6 small rectangular triangles. Label these small triangles according to their orientation following from $\mathcal{O}(F)$. The images of these triangles on $X(S)$ are thus in a one-to-one correspondence with the flag set $\mathscr{F}(S)$. One now proceeds with constructing a (continuous) conformal transformation of $\mathscr{C}(S)$ onto $\mathbf{P}^{1}$ by mapping each rectangular triangle onto the upper half plane, respectively lower half plane, according to their orientation such that the three angles are sent to $0,1, \infty$. This map is invariant under the equivalence relation $R$ so that it determines, at least in principle, the Belyi function. Hence, to a given equilateral triangulation one can assign the equilateral complex structure, obtained from a simplicial scheme $S$, provided this surface is $\overline{\mathbf{Q}}$-algebraic. It is important to realize that this map is independent of the metrical properties, i.e. of the size of the edges of the triangles! It is intuitively clear that the surface is necessarily algebraic: the only parameters entering the description of the map are the three branch points which are obviously algebraic. Thus we have assigned to a given triangulation a branched cover of the sphere, which is holomorphic (with respect to the equilateral complex structure) outside the three branch points. The conclusion is that this branched cover describes an arithmetic surface. That is, every planar graph of arbitrary topology (generated from a potential in matrix model, say) corresponds to an arithmetic surface described as a multiple cover of the sphere, branched over exactly three points. Even more is true: all arithmetic surfaces are be obtained in this way.

3.2. Strings from Triangulations. At this point it is useful to make contact with earlier attempts in [10] to assign a metric to a triangulated surface using planar diagram techniques of 't Hooft [31]. Like we did above one uses the dual graph of a triangulation and considers the propagator from the action (1.2) in a mixed coordinate-momentum representation. The vertices of the dual graph can be defined by the intersections of the medians in a triangulation. The edges of the dual graph have an interpretation in terms of momenta in a Mandelstam formulation of the string. Namely, introduce light-cone coordinates $X^{ \pm}=\frac{1}{\sqrt{2}}\left(X_{D-1} \pm X_{D}\right)$.

In a mixed momentum-coordinate representation it then follows that the action (1.2) gives a propagator for each edge $\langle i, j\rangle[10]$,

$$
\delta\left(P_{i j}^{+}-i\left(X_{i}^{+}-X_{j}^{+}\right) \exp \left(i P_{i j}\left(X_{i}^{-}-X_{j}^{-}\right)-\frac{1}{2}\left(X_{i}^{\perp}-X_{j}^{\perp}\right)^{2}\right) .\right.
$$


The integration over the coordinates $X_{i}^{-}$gives momentum conservation: $\sum_{i, j} P_{i j}^{+}=0$, where $P_{i j}$ denotes the momentum between two points $i, j$ of the triangulation. This conservation law implies that the momentum difference $P_{i j}^{+}$corresponds to an edge in the dual graph of the triangulation. After integrating over $X_{i}^{-}$the integrand reduces to

$$
\exp \left(-\sum_{i, j} \frac{1}{2}\left(X_{i}^{\perp}-X_{j}^{\perp}\right)^{2}\right)
$$

which almost looks like the propagator for a planar $S U(N)$-gauge theory

$$
\exp \left(-\sum_{i, j}\left|\frac{P_{i j}^{+}}{2 i\left(X_{i}^{+}-X_{j}^{+}\right)}\right|\left(X_{i}^{\perp}-X_{j}^{\perp}\right)^{2}\right),
$$

considered in [31, (5.1), p. 470] except for a crucial difference: in (3.14) there is a dynamical variable in front of the quadratic term in the exponent, whereas in (3.13) this is essentially the (non-zero) string constant $\alpha^{-1}$ (which we have put to unity). Since $\alpha^{-1}$ has the dimension of a mass it is not obvious how to obtain this parameter from the field theoretical "counterpart" $(3.13){ }^{6}$ This problem can be formulated more clearly as follows. Let us perform a "Wick rotation" and define a time coordinate $\tau_{i}=i X_{j}^{+}$. Thus one recovers the Mandelstam description of the world sheet: a vertex corresponds to the joining or splitting of string at a time $\tau_{i}$, each string piece having a width equal to the momentum difference $P_{i j}^{+}$(see Fig. 4). It is thus natural to consider the momentum at a dual vertex as a space coordinate $P_{i}^{+}=\sigma_{i}$.

The field equation for (1.2) is just the discrete Laplace equation

$$
\sum_{\langle i, j\rangle}\left(\tau_{i}-\tau_{j}\right)^{2}=0,
$$
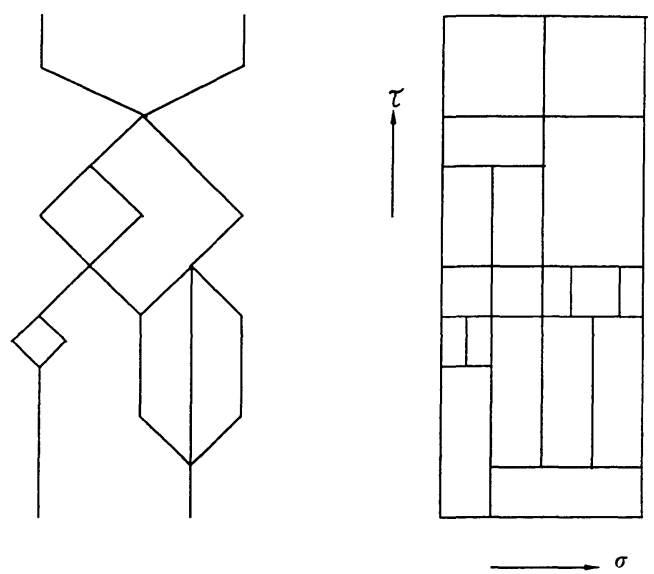

Fig. 4. The splitting and joining of strings

\footnotetext{
${ }^{6}$ I thank K. Bardakci for making me realize this
} 
where the sum runs over all edges in the triangulation. This implies a discrete version of Cauchy-Riemann's equation: ${ }^{7}$

$$
\tau_{i}-\tau_{j}=\sigma_{i}-\sigma_{j}
$$

The solution of (3.15-16) thus defines a local complex coordinate $z_{i}=\tau_{i}+i \sigma_{i}$ in each strip. The function $X_{i}^{\perp}$ is holomorphic in each strip with respect to this coordinate but ramifies at the vertices. It is not hard to see that the folding of a strip to a closed Riemann surface leads to the same compatibility condition on the vertices as explained above, that is the induced complex coordinate is exactly the coordinate (3.11) defined for the simplicial scheme $S$.

The problem mentioned above can now be restated as follows: In string theory the sum (1.3) over all triangulations is done for a fixed number of vertices, i.e. for fixed ratio

$$
\sum_{i j} \frac{1}{2}\left|\frac{P_{i j}}{\left(\tau_{i}-\tau_{j}\right)}\right| .
$$

This specifies the string constant $\alpha$ and hence the spectrum of the string. So far one was unable to impose this condition in a natural way starting from a triangulation, which thus prevents one to "derive" strings from triangulations (see [31] for example).

However, in our approach one can do this consistently using the theorem in the previous section, which relates a triangulation with a Riemann surface over a specific number field. In Sect. 4 it is shown that the condition of fixed area corresponds to fixing the number field in the expansion (2.42). This extra information (which is absolutely essential) was not explored in the attempts in [10]. Ahead of the analysis presented in the rest of this section, we thus arrive at an important conclusion:

"the original summation over metrics can be replaced by a summation over equilateral triangles corresponding to $\overline{\mathbf{Q}}$-algebraic points in the moduli space."

3.3. Triangulations of Genus $g$ and Ramified Covers of the Sphere. The tetrahedron discussed in Subsect. 3.2 corresponds to a triangulation of the sphere. Since any $\mathbf{P}^{1}$ is algebraic, we expect to have many inequivalent triangulations of it all corresponding to different finite extensions of $\mathbf{Q}$. The most obvious examples consist of the regular Platonic solids of which the tetrahedron is just an example. These triangulations of the sphere have symmetry groups that are subgroups of $S O(3)$, and consequently, the realization $|S|$ can be embedded into a three dimensional sphere. This is rather special, as a generic genus zero triangulation with realization $|S|$ has no such symmetry. Below we will outline a technique by which one can obtain in a few simple examples in genus zero and one the Belyi function and the number field. The discussion presented below does not solve the general problem of determining the Belyi function, it rather gives some insight why the generic problem is quite involved. Despite its limited use for this problem,

\footnotetext{
7 This construction was explained to me by V. Kazakov
} 
we shall see in the following subsection that the results below are of help in understanding the summation over triangulated surfaces.

Our technique is based on a simple fact: any genus $g$ equilateral triangulation can be described by a ramified covering of the sphere with $n$ distinguished points. This is allowed since we already showed that every open neighborhood of a vertex is biholomorphic equivalent to a punctured disc. If the center of the disc does not correspond to a point on the surface it defines a puncture, otherwise it is a regular distinguished point.

We fix the number of punctures to be $n$. Thus we are interested in an $n$-punctured sphere which we will describe by $X=\mathbf{C}-\left\{w_{1}, \ldots, w_{n}\right\}$, where $w_{i}$ denotes the location of the $i^{\text {th }}$ puncture. As is well known such a surface is uniformized by a Fuchsian group $\Gamma$ :

$$
X \cong H / \Gamma
$$

where $H$ is the upper-half plane and $\Gamma$ is a torsion-free subgroup in $P S L_{2}(R)$. Together with this one has the map $J: H \rightarrow X$ which is automorphic with respect to $\Gamma$, i.e. $J(\gamma z)=J(z)$ for all $\gamma \in \Gamma$. One can choose a set of parabolic generators $S_{1}, \ldots, S_{n}$ satisfying the relation

$$
S_{1} S_{2} \cdots S_{n}=1
$$

and which have the property that their fixed points denoted by $z_{1}, \ldots, z_{n} \in \mathbf{R} \cup\{\infty\}$ project onto $w_{1}, \ldots, w_{n}$. The map $J$ has an inverse $J^{-1}: X \rightarrow H$ which is a meromorphic function on $H$ of which the branch points are related with the linear fractional transformations in $\Gamma$. It gives rise to a so-called projective or uniformizing connection via the Schwarz differential equation:

$$
S\left(J^{-1}\right)=T_{X}
$$

where $T_{X}$ is known to be a rational function on $X$ :

$$
T_{X}(w)=\sum_{i=1}^{n-1}\left(\frac{1}{2\left(w-w_{i}\right)^{2}}+\frac{c_{i}}{w-w_{i}}\right) .
$$

The unknown parameters $c_{i}$, are the so-called accessory parameters of the Riemann surface $X$ and are in one-to-one correspondence with the parabolic fixed points and hence with the punctures $w_{i}[32,13]$. The differential $T_{X}(w) d w^{2}$ is a projective connection on $X$. It is referred to as the connection corresponding to the uniformization $J$.

Of course, one may choose three points at will, so we let $w_{1}=0, w_{2}=1, w_{3}=\infty$. If the point $w=\infty$ is a branch point of order $v$, the asymptotic expansion of $T_{X}$ reads

$$
T_{X}(w)=\frac{1}{2} \frac{\left(1-v^{-2}\right)}{w^{2}}+\sum_{j=0}^{\infty} a_{j} w^{j-3}
$$

Now let us consider the case of three punctures located at $w_{1}=0, w_{1}=1$, $w_{3}=\infty$. The function $T_{X}$ is explicitly known in this case see e.g. [32]. We denote it by $\mathscr{T}$ :

$$
\mathscr{T}=\frac{1}{2} \frac{\left(1-v_{1}^{-2}\right)}{w^{2}}+\frac{1}{2} \frac{\left(1-v_{2}^{-2}\right)}{(w-1)^{2}}+\frac{a}{w}+\frac{b}{(w-1)}
$$


with the constants $a$ and $b$ given by

$$
a=-b=\frac{1}{2}\left(1-v_{1}^{-2}-v_{2}^{-2}-v_{3}^{-2}\right) .
$$

The asymptotic formula (3.22) is true provided we take $v_{3}=v$. The numbers $v_{i}$ are the ramification indices of the points $w_{i}$.

The function $T_{X}$ for $n$-punctures involves the accessory parameters $c_{i}$, but contains also the term $\mathscr{T}$ :

$$
T_{X}=\mathscr{T}+\sum_{j=4}^{n} \frac{w_{j}\left(w_{j}-1\right)}{w(w-1)\left(w-w_{j}\right)}\left(\frac{\left(1-v_{j}^{-2}\right)}{2\left(w-w_{j}\right)}+c_{j}\right) .
$$

It is not hard to see that the parameters $c_{j}$ can be expressed as residues

$$
c_{j}=\frac{1}{w_{j}\left(w_{j}-1\right)} \operatorname{Res}_{w_{j}} w(w-1) T_{X}(w) d^{2} w, \quad j=4, \ldots, n,
$$

where $\operatorname{Res}_{w_{j}}$ denotes the residue at $w=w_{j}$. Summarizing, we have associated to the surface $X=H / \Gamma$ a function $T_{X}$ which ramifies at exactly the $n$ points corresponding to the parabolic fixed points of $\Gamma$. This is of course not the Belyi function, but let us see how the problem of determining the Belyi function can be solved in a simple example using the above.

In general may groups that uniformize $X$ and generically the function $T_{X}$ is not even unique for a given uniformization. Things are a little bit better if one insists on a Fuchsian uniformization, i.e. of the form discussed above: In this case the function $T_{X}$ is in one-to-one correspondence with $\Gamma$.

In [32] a useful formula is derived expressing the relation of the functions $T_{X}$ for two equivalent Fuchsian uniformizations of $X$. The result we need can be formulated as follows. Let $D_{1}$ and $D_{2}$ be two domains in the complex plane on which the groups $\Gamma_{1}, \Gamma_{2}$ act properly discontinuously. Assume a given covering $f: D_{1} \rightarrow D_{2}$ inducing a (surjective) homomorphism $f^{*}: \Gamma_{1} \rightarrow \Gamma_{2}$ such that

$$
f \circ \gamma=f^{*}(\gamma) \circ f \quad \forall \gamma \in \Gamma_{1} .
$$

The map $f$ induces a conformal map $F: D_{1} / \Gamma_{1} \rightarrow D_{2} / \Gamma_{2}$ such that the following diagram is commutative:

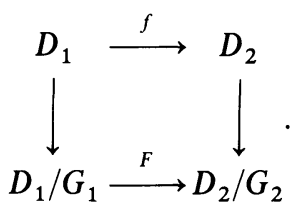

Associated with each projection $J_{i}, i=1,2$ one has a projective connection $T_{X}^{(1)}\left(z_{1}\right) d z_{1}^{2}$, respectively $T_{X}^{(2)}\left(z_{2}\right) d z_{2}^{2}$, where $z_{i}, i=1,2$ are local coordinates on $D_{i} \Gamma_{i}$. Using $f$ and properties of the Schwarz derivative it follows that

$$
T_{X}^{(1)}\left(z_{2}\right) d z_{2}^{2}=\mathscr{S}\left(f^{-1}\right) \text {. }
$$

In other words, $T_{X}^{(1)}$ is defined on $D_{2} / \Gamma_{2}$ only when the branch cuts on $D_{2}$ of $f^{-1}$ are related by a $P S L_{2}(\mathbf{R})$ transformation. (Since then $\mathscr{S}\left(f^{-1}\right)=0$.) This result turns out to be very useful for our purposes. Namely, we recall that the $2 n+2$-punctured sphere can be alternatively described by a hyperelliptic curve of genus $n$. So we 


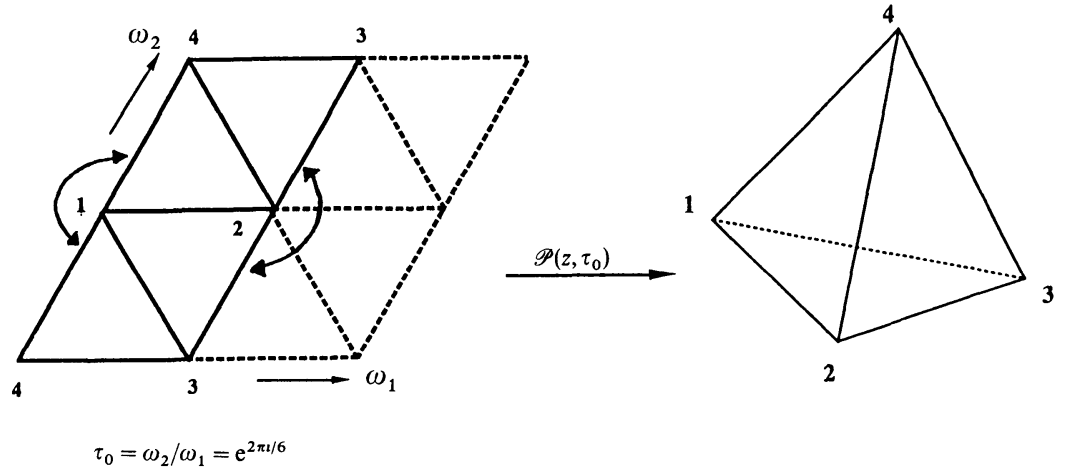

Fig. 5. Glueing the tetrahedron using the Weierstrass $\mathscr{P}$-function

have at least two equivalent uniformizations. The function that relates the two corresponding projective connections will be related to the Belyi-function.

To show this, we return to the tetrahedron example consisting of four triangles depicted in Fig. 5. Let $\omega_{1}$ and $\omega_{2}$ be two complex numbers and form the set

$$
\Omega=\left\{z \in \mathbf{C} \mid z \neq n_{1} \omega_{1}+n_{2} \omega_{2}, \forall n_{1}, n_{2} \in \mathbf{Z}\right\} .
$$

Let

$$
\phi: H \rightarrow \Omega
$$

be a covering of $\Omega$ by the upper-half plane. Next we introduce two groups. $\Gamma_{1}$ is generated by $\gamma_{1}(z)=z+\omega_{1}$ and $\gamma_{2}(z)=z+\omega_{2}$. This group acts on $\Omega$ and it is easy to see that the quotient $\Omega / \Gamma_{1}$ uniformizes a once punctured torus. The second group $\Gamma_{2}$ is generated by $\gamma_{1}, \gamma_{2}$ and $\gamma_{3}(z)=-z$. The group $\Gamma_{2}$ acts on $\Omega$ as well and it follows that $\Omega / \Gamma_{2}$ uniformizes a four-punctured sphere. Let $\mathscr{P}(\tau, z)$ be the Weierstrass $P$-function. This is an elliptic function depending on the modular parameter $\tau \in \Omega / \Gamma_{1}$ and the complex coordinate $z$. $\mathscr{P}$ induces a surjective map

$$
\Omega / \Gamma_{1} \rightarrow \Omega / \Gamma_{2}
$$

branched over $\infty$ and the three points $e_{1}=\mathscr{P}\left(\omega_{1} / 2\right), e_{2}=\mathscr{P}\left(\omega_{2} / 2\right), \mathscr{P}\left(\frac{\omega_{1}+\omega_{2}}{2}\right)$.

From the general result explained above it follows that the two uniformizing connections $T_{\Omega / G_{1}}$ and $T_{\Omega / G_{2}}$ are related by the Schwarzian derivative $\mathscr{S}\left(\phi^{-1}\right)$, which is unique up to a $P S L_{2}(\mathbf{R})$ transformation, and hence independent of the representation of $\phi$. The uniformizing connection for the once punctured torus is known to be, [33]:

$$
T_{\Omega / \Gamma_{1}}=\mathscr{S}\left(\phi^{-1}\right)=\frac{1}{2}(\mathscr{P}(\tau, z)+c(\tau)),
$$

where $c(\tau)$ is the accessory parameter of the once punctured torus with ramification index $v=1$. Using properties of the Weierstrass $\mathscr{P}$-function one may show that it has the following scaling behavior:

$$
c(\lambda \tau)=\frac{1}{\lambda^{2}} c(\tau)
$$


From this one may deduce that under $\tau \rightarrow \tau^{\prime}=\frac{a \tau+b}{c \tau+d}$ the accessory parameter transforms as

$$
c\left(\tau^{\prime}\right)=(c \tau+d)^{2} c(\tau) .
$$

Now, let $J: \Omega \rightarrow \Omega / \Gamma_{2}$ be the projection associated with the group $\Gamma_{2}$. In fact we see that $J=\mathscr{P} \circ \phi$, thus implying that $T_{\Omega / \Gamma_{2}}$ can be written as a function of $z$ as

$$
T_{\Omega / \Gamma_{2}}(z)=\mathscr{S}\left(J^{-1}(z)\right)=\mathscr{S}\left(\phi^{-1}(\xi)\right)\left(\frac{d \xi}{d z}\right)^{2}-\mathscr{S}(\xi(z)),
$$

where $\xi \equiv \mathscr{P}^{-1}(z)$ and $\mathscr{S}(f(z))$ denotes the Schwarzian derivative of $f$ with respect to $z$. The right-hand side can be computed in various ways, see e.g. [32]. The result reads

$$
\begin{aligned}
T_{\Omega / \Gamma_{2}}(z)= & \frac{3}{8}\left\{\frac{1}{\left(z-e_{1}\right)^{2}}+\frac{1}{\left(z-e_{2}\right)^{2}}+\frac{1}{\left(z-e_{3}\right)^{2}}\right\} \\
& -\frac{1}{4}\left\{\frac{1}{\left(z-e_{1}\right)\left(z-e_{2}\right)}+\frac{1}{\left(z-e_{1}\right)\left(z-e_{3}\right)}+\frac{1}{\left(z-e_{2}\right)\left(z-e_{3}\right)}\right\}
\end{aligned}
$$

with $z$ a coordinate on $\mathbf{P}^{1}$. This is the projective connection for the punctured sphere branched over $e_{i}, i=1,2,3$ and $\infty$ with ramification indices $v_{i}=v=2$. Using the expression for the accessory parameter in terms of a residue, one may recast this (with $e_{1}=0, e_{2}=1, e_{3}=\infty$ ) into

$$
\begin{aligned}
T_{\Omega / \Gamma_{2}}= & \frac{3}{8} \frac{z^{2}-z+1}{z^{2}(z-1)} \\
& +\frac{w(w-1)}{z(z-1)(z-w)}\left\{\frac{3}{8} \frac{1}{z-w}+\frac{1}{8} \frac{2 w-1}{w(w-1)}\right\}
\end{aligned}
$$

so that the accessory parameter for the 4-punctured sphere reads

$$
c(w)=\frac{1}{8} \frac{2 w-1}{w(w-1)}
$$

with $w$ the location of the $4^{\text {th }}$ puncture. We thus see that the determination of the accessory parameter for the 4-punctured sphere is equivalent with the determination of the accessory parameter of the once-punctured torus.

Now, let us take the location of this puncture such that it corresponds to the fourth vertex of a regular tetrahedron. In our coordinates this means $w=\exp 2 \pi i / 3$. This fixes the value of the modular parameter in the Weierstrass function to be $\tau=\exp 2 \pi i / 6$. For this value of the modular parameter the Weierstrass function is easily seen to glue the four equilateral triangles in the complex plane into a regular tetrahedron. See Fig. 5. The elliptic curve described by this Weierstrass function is easily computed: it has the form

$$
y^{2}=x^{3}+1,
$$

and is defined over the quadratic imaginary extension $\mathbf{Q}\left(e^{2 \pi i / 3}\right)$ of $\mathbf{Q}$. We have thus found that the Weierstrass $P$-function explicitly describes the genus zero 
triangulation corresponding to the tetrahedron. Along a different way this result has also been obtained by M. Bauer and C. Itzykson [34].

The Weierstrass $P$-function is not the Belyi function for this triangulation. However, the result above implies that this function must be an algebraic function of the $\mathscr{P}$-function. At this point we recall the general construction of the Belyi function in [6] discussed in Subsect. 3.1 and the fact that the triangulation has a symmetry group corresponding to the modulary group $\Gamma / \Gamma(3) \cong A_{4}$ which is a discrete subgroup of the three dimensional rotation group $S O(3) .{ }^{8}$ If we draw the medians in each of the four equilateral triangles and assign to each of the points the values of the function $\mathscr{P}(z)$ we find that the Belyi function is six sheeted cover of the sphere uniformized by $H / \Gamma(3)$. Hence the Belyi function corresponds to the $\mathscr{J}_{3}$-function on the fundamental domain of $\Gamma(3)$ which parametrizes a six-fold cover of $\mathbf{P}^{1}$,

$$
J_{3}=\frac{4}{27} \frac{\left(\lambda^{2}-\lambda+1\right)^{3}}{\lambda^{2}(\lambda-1)^{2}}, \quad \lambda(\tau)=\frac{\vartheta_{2}^{4}(\tau)}{\vartheta_{3}^{4}(\tau)} .
$$

At first one may wonder by the Weierstrass $P$-function is related to a genus zero triangulation since it is an elliptic function hence intuitively related to a genus one surface. The reason is as follows. The complete genus one curve corresponds to a map $\mathbf{C} \rightarrow \mathbf{C}^{2}$, defined by $z \rightarrow\left(\mathscr{P}(z), \mathscr{P}^{\prime}(z)\right)$, having Weierstrass equation

$$
y^{2}+a_{1} x y+a_{3} y=x^{3}+a_{2} x^{2}+a_{4} x=a_{6},
$$

where $y=\mathscr{P}^{\prime}(z)$ and $x=\mathscr{P}(z)$. In the construction above one projects this curve back to the $\mathbf{C}$ by ignoring essentially the $\mathscr{P}^{\prime}(z)$ part, which forces $a_{1}=a_{2}=a_{3}=a_{4}=0$. In other words: one forgets about one of the sheets of the cover.

From this example one may easily obtain genus one triangulations by considering the full curve (3.40) in $\mathbf{C}^{2}$ describing a torus. It describes a triangulation in genus one, shown in Fig. 5. This fact is a simple consequence of the well known fact that the Picard $\lambda$-function $\lambda=\frac{\vartheta_{2}^{4}(\tau)}{\vartheta_{3}^{4}(\tau)}$ is a rational function. One may show this explicitly using relations among theta-functions [36].

The above example is an illustration of a much deeper theorem proved by Belyi, on finite extensions with given Galois group. The theorem quoted in Subsect. 3.1 is in fact a corollary of this result. We will not discuss this theorem

${ }^{8}$ The modulary groups that we are interested in arise in the context of genus zero subgroups of the modular group. The simplest examples of genus zero subgroups $\Gamma$ are (apart from the full modular group $\Gamma=P S L_{2}(\mathbf{Z})$ itself) the inhomogeneous principal congruence subgroups $\Gamma(N)$ with $N \leqq 5$ defined by the matrices in $P S L_{2}(Z)$ obeying the condition:

$$
\left(\begin{array}{ll}
a & b \\
c & d
\end{array}\right)=\left(\begin{array}{ll}
1 & 0 \\
0 & 1
\end{array}\right) \quad \operatorname{Mod} N
$$

The modulary group $\Gamma_{N} \equiv P S L_{2}(\mathrm{Z}) / \Gamma(N)$ corresponds to the symmetry group of the triangulation of the sphere corresponding to regular Platonic polyhedrons. The surfaces $X$ are parametrized by a function $\mathscr{J}(z), z \in \mathbf{C}$ being the coordinate on $\mathbf{P}^{1}$. These coordinates are well known in the literature [35] 
here, but mention a few facts related to it, which may be of use for the continuum theory. The tetrahedron has the (obvious) automorphism group $A_{4}$ of 12 elements. The result of Belyi provides a construction which assigns a surface to this polyhedron described by a cover of the sphere which is defined over a finite extension having Galois group which is exactly the symmetry of the triangulation. Roughly speaking one thus concludes that planar graphs of arbitrary topology in fact correspond to surfaces having an "unusual" symmetry given by the Galois group of the finite extension over which it is defined! In case of the other Platonic polyhedrons we can be more explicit on this point. The symmetries of these polyhedrons correspond to finite subgroups of $S O(3)$. In order to have a Galois group interpretation of these subgroups we need a slightly different description. Namely, let $F$ be a number field (of characteristic $l$, and let $G$ be a finite subgroup of (the Chevalley group) $G L_{2}(F)$ (of order prime to $l$ ). Let $H$ be the image of $G$ in $P G L_{2}(F)$. One then has the following cases [38]

1. $H$ is cyclic;

2. $H$ is dihedral (i.e. it contains a cyclic subgroup of index 2);

3. $H$ is isomorphic to $A_{4}, S_{4}$ or $A_{5}$.

It follows from some relatively straightforward calculations that the groups $G$ indeed correspond to Galois groups of finite extensions of $\mathbf{Q}$ over which the arithmetic curves are defined associated with the polyhedrons (upon application of Belyi's theorem).

In general, however, a genus zero triangulation does not have a three dimensional rotation symmetry, and in general it cannot be embedded in a three dimensional space. That is, other Chevalley groups (and possible finite subgroups) will occur as Galois groups of the associated curves. Such curves will be, however, no longer described by a single equation but rather by a set of equations all having coefficients and solutions in the same finite extension. In the example above, we just needed one equation. It is only for the Platonic polyhedrons, that a single equation suffices (with in addition some projection). The situation for higher genus surfaces is even more difficult as for generic genera the number of equations necessary to describe a triangulation of the same topology is over determined, i.e. the co-dimension is lower than the number of equations! A generic arithmetic surface has no automorphisms. At this point it is amusing to recall that in [39] it was shown surfaces with automorphisms may still be very important. Namely, it was shown there that high energy scattering in critical string theory (i.e. for large orders in perturbation theory) the main contribution comes from surfaces having at least a cyclic symmetry. These surfaces are just examples of arithmetic surfaces described by a single algebraic equation! Of course, this is only a superficial suggestion indicating that the continuum theory has a tendency to single out "symmetric" arithmetic surfaces.

It is now clear that in the DT approach to string theory we are interested in the distribution of arithmetic surfaces in the moduli space of genus $g$ surfaces. This density is related to the modular height as the saw in the previous section. Despite the difficulty of determining the Belyi function explicitly, we can be more precise on the relation between the modular height function and triangulations of surfaces. Let us fix a given triangulation and write the corresponding surface as $X=H / \Gamma$ 
for some Fuchsian group $\Gamma$ (assuming that a Fuchsian uniformization of $X$ exists $^{9}$ ). Correspondingly we have the map $J$ and its inverse $J^{-1}$ as we discussed before. Since we started from a triangulation, there is a fixed number field $K$ over which $X$ is defined. As follows from the properties of the Belyi function, $K$ specifies the number of vertices necessary for the triangulation. The number of sheets of the cover defined by the Belyi function is a measure for the number of vertices. All possible triangulations with the same number of vertices (and for fixed topology) thus correspond to different $K$-algebraic points in the moduli space of $X$. Each different point has its own uniformizing group $\Gamma$, which determines the location of the accessory parameters, and hence the "shape" of the triangulation. The global structure of the triangulation is thus determined by its topology and the number field, whereas the "local" properties follow from the uniformization.

The partition function on the moduli space $\mathscr{M}$ of $X$ defined over $K$ thus corresponds to a function measuring the distance between any two $K$-algebraic points, but this is in fact the modular height function. In the next section we will see that in the case of triangulations of the sphere the relation between the modular height and the geometry of the moduli space becomes more explicit.

We close this subsection with a side remark on the metric on the surface $X$ compatible with the complex structure (3.11). We would like to show that the uniformization of the Riemann surface $X$ can be used to show that a suitable surface element measures the density of squares in Fig. 4. Namely, on $H$ one has the Poincaré metric

$$
\lambda_{H}(z) d z=\frac{d z}{1-|z|^{2}}
$$

which induces a metric on $H / \Gamma($ since $\Gamma \subset \operatorname{Aut}(H))$,

$$
\lambda_{H / \Gamma}(Z) d Z=\lambda_{H}(z) d z .
$$

The metric on $H / \Gamma$ will have singularities at the points of ramification, but is still integrable over $H / \Gamma$. For example, at a vertex we have the coordinate (3.11), so that the metric behaves at the ramification points as

$$
\lambda_{H / \Gamma}(Z)=\frac{|Z|^{v-1} d Z}{v\left(1-|Z|^{2 / v}\right)}
$$

with $v=n / 6$ the ramification index at the vertex. This metric is clearly integrable. It is not hard to show, using a discrete Gauss-Bonnet theorem as in [37] that the area of $H / \Gamma$ is given by

$$
\operatorname{Area}(H / \Gamma)=2 \pi\left((2 g-2)+\sum_{k=1}^{N}\left(1-v_{k}^{-1}\right)\right),
$$

where $N$ is a number of vertices. Now recall that the curvature represented at each vertex is given by $R_{i}=\pi\left(v_{i}^{-1}-1\right)$. Thus we recover that $\lambda(Z)$ indeed corresponds to the density of squares in Fig. 4.

\footnotetext{
${ }^{9}$ In general this will not be so: one needs a Shottky group (i.e. a particular Kleinian group). The argument below still goes through except for a few technical modifications
} 


\section{The Sum over Triangulated Surfaces}

Let us finally return to the problem of relating the volume of the lattice formed by the algebraic points in the moduli space with the discretized string integral (1.3) for equilateral triangulations. We will discuss those rational points that define curves, for which the moduli space is $\mathscr{M}_{0, n}$ of a sphere with $n$-punctures. We shall argue that (2.42) when restricted to hyperelliptic curves equals (1.3) in the case the triangulations are described by some finite cover of the sphere. In order to obtain this result we will use a result in [13] which relates the Kähler form on $\mathscr{M}_{0, n}$ with the accessory parameters of the sphere. Subsequently, we discuss a limit to all algebraic points which bears some resemblance with the double scaling limit in a matrix theory.

The geometry of the moduli space $\mathscr{M}_{0, n}$ is conveniently described by the Weil-Petersson (WP) Kähler metric, defined by a Hermitian innerproduct for holomorphic quadratic differentials using the hyperbolic line element on the Riemann surface. Namely for $\phi$ a holomorphic quadratic differential and $\lambda^{2}$ the hyperbolic line element (i.e. the constant curvature metric) on the Riemann surface $X$ we form the Beltrami differential $\bar{\phi} \lambda^{-2}$ and define the Weil-Petersson inner product as

$$
\langle\phi, \psi\rangle_{\mathrm{wp}}=\int_{X}\left(\phi \lambda^{-2}\right)\left(\overline{\bar{\psi} \lambda^{-2}}\right)=\int_{X} \bar{\phi} \psi \lambda^{-2}
$$

Note that this definition makes use of the (unique) constant curvature metric compatible with the given complex structure on $X$.

The WP metric arises naturally in the context of accessory parameters as was shown in [13]. This is a consequence of the fact that the Teichmüller space of an $n$-punctured sphere can be mapped into an $n$-dimensional complex vector space $W_{n}$ defined by

$$
W_{n}=\left\{\left(w_{1}, \ldots, w_{n}\right) \in \mathbf{C}^{n} \mid w_{j} \neq 0,1 ; w_{i} \neq w_{j} \leftrightarrow i \neq j\right\} .
$$

This map plays an essential role below, so let us explain this in a little more detail. (We use the notation of [13].) Let $\Gamma$ be the Fuchsian group uniformizing $X$. Then the Beltrami differential $\mu=\bar{\phi} \lambda^{-2}$ introduced above satisfies $\mu(\gamma z) \overline{\gamma^{\prime}(z)} / \gamma^{\prime}(z)=\mu(z)$ for all $\gamma \in \Gamma$. ( $z$ is the coordinate on the upper half plane.) Now, for each $\mu$ the Beltrami equation gives rise to a function $f_{\mu}(z)$ which in turn gives rise to a Fuchsian group $\Gamma_{\mu}=f_{\mu} \Gamma f_{\mu}^{-1}$ which is of the same type as $\Gamma$, that is $\Gamma_{\mu}$ is generated by $f_{\mu} S_{i} f_{\mu}^{-1}$ with $i=1, \ldots, n$. In other words: for each conformal equivalence class labelled by a Beltrami differential we have a representation $\rho_{\mu}$ of $\Gamma$, i.e. $\Phi: \Gamma \rightarrow P S L_{2}(\mathbf{R})$ defined by $\gamma \rightarrow f_{\mu} \circ \gamma \circ f_{\mu}^{-1}$. Denote the corresponding map $\mu \rightarrow \rho_{\mu}$. It is well known that the set of all equivalence classes of $\rho_{\mu}$ gives a model of Teichmüller space.

Now embed the surface $X$ into $\mathbf{C} \cup\{\infty\}$, and let $J_{\mu}$ be the projection map $H \rightarrow X \cong H / \Gamma_{\mu}$ fixing the points $0,1, \infty$. Furthermore, define the map $w^{\mu}$ as the composition $w^{\mu}=J_{\mu} \circ d_{\mu}\left(z_{i}\right)$ with $z_{i}$ one of the parabolic fixed points of $\Gamma$. We then define the map $\Psi: T_{0, n} \rightarrow \mathbf{C}^{n-3}$ by

$$
(\Psi \circ \Phi)(\mu)=\left(w_{1}^{\mu}, \ldots, w_{n}^{\mu}\right) \in \mathbf{C}^{n-3} .
$$

As is shown in $[13,32]$ this map defines a complex analytic cover of $T_{0, n}$ by $W_{n}$, 
i.e. the image of (3.48) in $\mathbf{C}^{n-3}$ is the set $W_{n}$, and locally it is an diffeomorphism. On the space $W_{n}$ we have of course the action of the symmetric group $\mathrm{Sym}_{n}$ acting on the $n$ punctures. The factor space $W_{n} / \mathrm{Sym}_{n}$ is isomorphic to the moduli space $\mathscr{M}_{0, n}$. The most notable feature of the map is that it is a finite cover.

On $W_{n}$ we have the canonical flat metric. The result we need from [13] is the fact that the Jacobian of the transformation relating the WP metric $T_{0, n}$ with the metric on $W_{n}$ is given by the Liouville action functional for the conformal factor $\sigma$, evaluated at its extremum, and the accessory parameters on $X$ lifted to $T_{0, n}$. The Liouville action enters the problem, via its field equation and its relation to the uniformization of the surface $X$. Namely recall that the unique hyperbolic metric on $X$ is obtained from the Poincare metric $\frac{d z d \bar{z}}{(\operatorname{Im} z)^{2}}$ on the upper-half plane
projected on $X$ which is of the form

$$
\exp (\sigma(w))|d w|^{2}, \quad \exp (\sigma(w))=\frac{\left|J^{-1}(w)\right|^{2}}{\left(\operatorname{Im} J^{-1}(w)\right)^{2}},
$$

where $\sigma(w)$ is the solution $\partial_{w} \bar{\partial}_{\bar{w}} \sigma=\frac{1}{2} \exp \sigma$. With suitable asymptotics, this solution is unique. The formula for $T_{X}$ can be written in terms of the chiral part of the stress-energy tensor of the Liouville theory:

$$
T_{X}=\partial_{w}^{2} \sigma-\frac{1}{2}\left(\partial_{w} \sigma\right)^{2},
$$

obtained from the action

$$
S[\sigma]=\int_{X}\left(\left|\partial_{w} \sigma\right|^{2}+\exp \sigma(w)\right) d w \wedge d \bar{w} .
$$

The map (4.3) can be used to define the field $\sigma(w)$ on $W_{n}$ so that (4.5) is defined on $W_{n}$ as well. It is shown in [13] that the accessory parameters of $X$ (present in the function $T_{X}$ ) can be obtained from the Liouville action:

$$
c_{j}\left(w_{1}, \ldots, w_{n}\right)=\frac{\partial S[\sigma]}{\partial w_{j}},
$$

with $\sigma$ satisfying Liouville's equation. Of course, the accessory parameters transform under $\Gamma$. This is used to show that upon taking a second derivative one obtains a volume form on $W_{n}$ which in fact can be pulled back to the Weil-Petersson Kähler form on $T_{0, n}$ :

$$
\frac{\partial c_{j}}{\partial w_{k}}=\frac{\partial^{2} S[\sigma]}{\partial w_{j} \bar{\partial} \bar{w}_{k}} d w_{j} \wedge d \bar{w}_{k}=w_{j k},
$$

where $\omega_{j k}$ denotes the Weil- Petersson Kähler form on $T_{0, n}$ projected on $W_{n}$. Upon modding out the action of the symmetric group one obtains the WP form on the moduli space of $n$-punctured spheres. Thus we see that the Liouville action (4.5) corresponds to the Jacobian of the transformation (4.3).

The relation between the accessory parameters and the WP metric is useful for the following reason. It follows from [12] that there is a relation between the cohomology class of the WP-Käler form with the (metrized) line bundle det $\pi_{*} \omega_{X / M}$ which played a central role in the Polyakov measure. More precisely, the WP class is isometric to some power of the determinant line bundle $\operatorname{det}_{*}^{\prime} \omega_{X / \mathscr{M}}$ equipped 
with the Green's metric (with respect to the hyperbolic metric on $X$ ): ${ }^{10}$

$$
\frac{1}{24 \pi^{2}} \omega_{\text {w.p. }}=\operatorname{det} \pi_{*} \omega_{X / \mathscr{M}} \text {. }
$$

Let us now analyze the behavior of (2.42) for large extensions $K$. It is useful in this context to recall that the height $h(X)$ is a distance function, measuring the logarithmic distance to the closest $K$-algebraic point in $\mathscr{M}$. Since we have to take the square of the expression (2.42) for the complete partition function we conclude that $\exp h(X)$ measures the surface density of $K$-rational points in the moduli space. However, we must take into account a symmetry factor for each rational point as well as corresponding to the symmetry group of the triangulation. In order to analyze the behavior of (2.42) for large extensions we therefore rewrite (2.42),

$$
\lim _{k \rightarrow \infty} \lambda_{g}^{\chi} \mathcal{N}(k, \chi) \exp (\mu h(X))
$$

where we introduced a multiplicative (complex) parameter $\mu$ in the exponent to improve convergence; eventually we have to take the limit to some critical value.

The object $\mathscr{N}(k, \chi)$, depending on the degree of the extension $k \equiv[K: \mathbf{Q}]$ and the Euler number $\chi=2 g-2$ of the surface, measures the number of algebraic points over $K$ in the moduli space of $X$. Now, in order to find the asymptotics of $\mathscr{N}(k, \chi)$ we make two assumptions which in fact are generally believed to be true but for which there is no rigorous proof:

1. the number of vertices in the triangulation necessary to describe a curve defined over $K$ grows with $k$ ! in the limit of large extension;

2 . the area density element $A$ of $K$-algebraic points on the moduli space grows as a power equal to the euler number of the surface. Thus, for low genus the number of algebraic points is large, while for high genus the number is low.

Let us fix a large degree of extension, $k$, and consider only hyperelliptic surfaces. So, on a hyperelliptic curve over a large but fixed extension we need according to the assumption roughly $N=k$ ! points on the sphere to describe the associated triangulation. The number $\mathscr{N}(k, \chi)$ thus gives the correct weight of equilateral triangulation possible for $N$ points:

$$
\mathscr{N}(k, \chi)=\sum_{i \in \mathscr{T}} \frac{1}{\left|G_{i}\right|},
$$

where $\mathscr{G}_{i}$ is the symmetry group of a triangulation labelled by $i \in \mathscr{T}$, as in (1.3). This quantity behaves for large $N$ as

$$
\mathscr{N}(N, \chi) \sim N^{\gamma \chi-1} e^{\mu_{c} N} C_{\chi}\left(1+\mathcal{O}\left(\frac{1}{N}\right)\right),
$$

\footnotetext{
10 This formula can be extended to the boundary of moduli space, in which case on has to add on the right-hand side the compactification divisor $\Delta$. This inclusion gives rise to a divergence in the metric on the right-hand side and correspondingly in the sum over all $\overline{\mathbf{Q}}$ algebraic points in (2.42). This divergence corresponds to the tachyon in the spectrum of the critical string [20]. In terms of triangulations such a singularity corresponds to a degenerate triangulation in which some of the vertices coalesce
} 
where the constants $\gamma$ and $C_{\chi}$ are universal. ${ }^{11}$ As will become clear below, we deliberately used the same notation for the constants as in matrix model analysis.

At this point we recall that the number vertices $N$ is at the same time the measure for the area $A$ of the triangulation. So the limit $N \rightarrow \infty$ corresponds in fact to a surface integral:

$$
\lim _{N \rightarrow \infty} \mathscr{N}(N, \chi) \cong C_{\chi} \int_{0}^{\infty} d A A^{\gamma \chi-1} e^{\mu_{c} A}
$$

We now want to substitute this result in (4.9). However, we have to reach a decision on what part of the height function we want to take. As can be seen explicitly from the expressions the height there is a relative sign difference between the contribution to the height from the finite places and the infinite places. This means that if one insists on a real constant $\mu_{c}$ one cannot control the sum for both parts simultaneously. So let us for the moment consider the part coming from the infinite places. Since the height as it enters in (2.42) describes a surface area as well, the large $N$ behavior of $(2.42)$ can be expressed as

$$
\mathscr{Z}_{g} \cong C_{\chi} \int_{0}^{\infty} d A A^{\gamma \chi-1} e^{-\left(\mu-\mu_{c}\right) A}=C_{\chi} \Gamma(\chi)\left|\mu-\mu_{c}\right|^{\gamma \chi} .
$$

The result suggests the definition of a double scaling limit, namely if one performs the summation over all genera the expression

$$
\mathscr{Z}=\sum_{\chi} \lambda^{-\chi \mathscr{Z}_{g}}=\sum_{\chi} \Gamma(\gamma \chi)\left|\frac{\mu-\mu_{c}}{\lambda}\right|^{\gamma \chi}
$$

is well defined provided the multiplicative constant on the right-hand side is kept fixed in the process of taking the limit. On the other hand one may consider the finite part of the height, and taking $-\mu$ as the constant in (2.42). This leads formally to the same scaling behavior. One may include both cases at the same time by taking a complex coefficient $\mu$. Then (2.42) behaves more like a Zeta-function which at least from the point of view of "computing volumes" is more comfortable, (see e.g. [9]). In matrix models one identifies the constant $\mu$ with the (bare) cosmological constant and is thus usually taken to be real.

Let us make a remark at this point. Note that because of the Gamma-function, (4.13) grows roughly with $(2 g)$ ! This growth behaviour is confirmed also by calculations done in the DT approach [41]. Recall, however, that our approach is geometrical, in the sense that it does not rely on a potential in a matrix model.

Acknowledgements. I wish to thank O. Alvarez, K. Bardakci, J. Distler, A. Giveon, V. Kazakov, E. Kiritsis, P. Mende and A. Polyakov for their interest and many helpful comments. I want to thank also B. Edixhoven for explaining some aspects of the theory underlying the result in [6]. Finally, I thank P. Nelson for bringing ref. [11] to my attention.

11 The constant $\mu_{c}$ depends, however, on the type of triangulation. We considered only equilateral triangles. For more general triangulations of a two dimensional surface the same asymptotic formula holds, but for different $\mu_{c}$ 


\section{References}

1. Kazakov, V.: Bilocal regularization of models of random surfaces. Phys. Lett. 150, 282 (1985); Kazakov, V., Kostov, I., Migdal, A.: Critical properties of randomly triangulated planar random surfaces. Phys. Lett. 157B, 295 (1985)

2. David, F.: Planar diagrams, two-dimensional lattice gravity and surface models, Nucl. Phys. B257 [FS14], 45 (1985); and: A model of random surfaces with non-trivial critical behaviour. Nucl. Phys. B257 [FS14], 543 (1985)

3. Brézin, E., Kazakov, V. A.: Exactly solvable field theories of closed strings. Phys. Lett. 236B, 144 (1990); Douglas, M., Shenker, S.: Strings in less than one dimension, Rutgers preprint RU-89-34. October 1989; Gross, D., Migdal, A.: Non-perturbative two dimensional quantum gravity. Phys. Rev. Lett. 64, 127 (1990)

4. Smit, D.-J.: String theory and the algebraic geometry of moduli spaces. Commun. Math. Phys. 114, 645 (1988)

5. Ueno, K.: Bosonic string and arithmetic surfaces, Kyoto University preprint, December 1986

6. Voedvodski, V., Shabat, G.: Equilateral triangulations of Riemann surfaces and curves over algebraic number fields. Sov. Math. Dokl. 39, 38 (1989)

7. Edixhoven, B.: The Polyakov measure and the modular height function. In: Proceedings of the Arbeitstagung on arithmetical algebraic geometry, Wuppertal 1987

8. Smit, D.-J.: Algebraic and arithmetic geometry in string theory. In: Proceedings of the 16-th colloquium on group theoretical methods in physics. Lecture Notes in Phys. vol. 313, pp. 515. Berlin, Heidelberg, New York: Springer 1988

9. Manin, Yu.: Reflections on arithmetical physics. In: The proceedings of the Poiana-Brasov school on strings and conformal field theory, 1987

10. Kostov, I.: Random surfaces, solvable lattice models and discrete quantum gravity in two dimensions, Saclay preprint S.Ph-T/88-196, 1988; Kazakov, V., Bulatov, D., Zamolodchikov, A.: unpublished

11. Levin, A., Morozov, A.: On the foundations of random lattices approach to quantum gravity, ITEP-preprint Moscow 1990

12. Harris, J., Mumford, D.: On the Kodaira dimension of curves. Invent. Math. 67, 23 (1982)

13. Zograf, P. Takhtajan, L.: Action of the Liouville equation is a generating function for the Weil-Petersson metric on the Teichmüller space. Funct. Ann. Appl. 19, 219 (1985) and: On the Liouville equation, accessory parameters and the geometry of Teichmüller space for Riemann surfaces of genus zero. Math. USSR Sbornik 60, 143 (1988)

14. Bogomolov, F.: Rationality of the moduli of hyperelliptic curves of arbitrary genus. In: Can. Math. Soc. Conf. 6, 17 (1986). (The original proof is due to P. Kacilo.)

15. Friedan, D., Shenker, S.: The analytic geometry of $d=2$ conformal field theory. Nucl. Phys. B281, 50 (1987)

16. Beilinson, A., Manin, Yu.: The Mumford form and the Polyakov measure in string theory. Commun. Math. Phys. 107, 359 (1986)

17. Alvarez-Gaumé, L., Bost, J., Moore, G., Nelson, P., Vafa, C.: Bosonization on higher genus Riemann surfaces. Commun. Math. Phys. 113 (1987)

18. Deligne, P., Mumford, D.: The irreducibility of the space of curves of given genus. Publ. I.H.E.S. 36, 75 (1969)

19. Mumford, D.: On the stability of projective varieties. L'Ens Math. 23, 39 (1977)

20. Belavin, A., Kniznik, V.: Algebraic geometry and the geometry of quantum strings. Sov. Phys. JETP 64, 214 (1986)

21. Arakelov, S.: Intersection theory of divisors on an arithmetic surface. Izv. Akad. Nauk. 38, 1179 (1974)

22. Faltings, G.: Calculus on arithmetic surfaces. Ann. Math. 119, 387 (1984)

23. Manin, Yu.: The partition function of the string can be expressed in terms of theta-functions. Phys. Lett. B172, 184 (1986)

24. Quillen, D.: Determinants of Cauchy-Riemann operators. Funkt. Anal. Appl. 19, 31 (1985)

25. Verlinde, E., Verlinde, H.: Chiral bosonization, determinants and the string partition function. Nucl. Phys. B288, 357 (1987)

26. Bismut, J., Freed, D.: The analysis of elliptic families: Dirac operators, eta-invariants and the holonomy theorem of Witten. Commun. Math. Phys. 107, 103 (1986) 
27. See for this result: Lang, S.: Fundamentals of Diophantine geometry, Chap. 5, Berlin, Heidelberg, New York: Springer 1983

28. Silverman, J.: Height and elliptic curves. In: Arithmetic geometry. Cornell, G., Silverman, J. (eds.) Berlin, Heidelberg, New York: Springer 1986

29. Morozov, A.: Explicit formulae for one, two, three and four loop string amplitudes. Phys. Lett. 184B, 173 (1977)

30. Belyi, G.: On Galois extensions of a maximal cyclotomic field. Math. USSR Izv. 14, 247 (1980)

31. 't Hooft, G.: A planar diagram theory for strong interactions. Nucl. Phys. B72, 461 (1974)

32. Kra, I.: Accessory parameters for punctured spheres, MSRI-preprint. Berkeley 1988; and: Automorphic forms and Kleinian groups. Reading, Mass.: Benjamin 1972

33. Keen, L.: Rauch, H., Vazques, A.: Moduli of punctured tori and the accessory parameter of Lamé's equation. Trans. Am. Math. Soc. 255, 201 (1975)

34. Itzykson, C.: Private communication

35. Fricke, R.: Lehrbuch der Algebra, Vol. 2, Braunsweig 1926

36. Mumford, D.: Tata lectures on theta, Vol. 1. Bonn: Birkhäuser 1983

37. Farkas, H.: Kra, I.: Riemann surfaces, G..T.M. vol. 71, Berlin, Heidelberg, New York: Springer 1980

38. Lang, S.: Introduction to modular forms. Berlin, Heidelberg, New York: Springer 1976

39. Gross, D., Mende, P.: String theory beyond the Planck scale. Nucl. Phys. B303, 407 (1988)

40. Wolpert, S.: On the homology of the moduli space of stable curves. Invent. Math. 118, 491 (1983)

41. Shenker, S.: Talk at the Cargèse Conference on 'String theory, 2D gravity and triangulated random surfaces', June 1990

Communicated by N. Yu. Reshetikhin 
